\title{
Resolving the Atomistic Modes of Anle138b Inhibitory Action on Peptide Oligomer Formation
}

\author{
Dirk Matthes, ${ }^{\dagger}$ Vytautas Gapsys, $^{\dagger}{ }^{\circ}$ Christian Griesinger, ${ }^{\ddagger}$ and Bert L. de Groot* ${ }^{* \dagger}$ \\ ${ }^{\dagger}$ Computational Biomolecular Dynamics Group, Department of Theoretical and Computational Biophysics, Max Planck Institute for \\ Biophysical Chemistry, Am Fassberg 11, 37077 Göttingen, Germany \\ ${ }^{\ddagger}$ Department of Structural Biology, Max Planck Institute for Biophysical Chemistry, Am Fassberg 11, 37077 Göttingen, Germany
}

Supporting Information

\begin{abstract}
The diphenyl-pyrazole compound anle138b is a known inhibitor of oligomeric aggregate formation in vitro and in vivo. Therefore, anle $138 \mathrm{~b}$ is considered a promising drug candidate to beneficially interfere with neurodegenerative processes causing devastating pathologies in humans. The atomistic details of the aggregation inhibition mechanism, however, are to date unknown since the ensemble of small nonfibrillar aggregates is structurally heterogeneous and inaccessible to direct structural characterization. Here, we set out to elucidate anle138b's mode of action using all-atom molecular dynamics simulations on the multi-microsecond time scale.

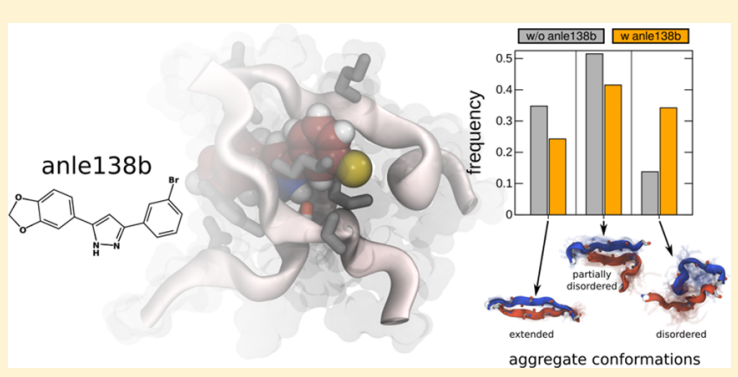
By comparing simulations of dimeric to tetrameric aggregates from fragments of four amyloidogenic proteins (A $\beta$, hTau 40 , hIAPP, and Sup35N) in the presence and absence of anle138b, we show that the compound reduces the overall number of intermolecular hydrogen bonds, disfavors the sampling of the aggregated state, and remodels the conformational distributions within the small oligomeric peptide aggregates. Most notably, anle138b preferentially interacts with the disordered structure ensemble via its pyrazole moiety, thereby effectively blocking interpeptide main chain interactions and impeding the spontaneous formation of ordered $\beta$-sheet structures, in particular those with out-of-register antiparallel $\beta$-strands. The structurally very similar compound anle234b was previously identified as inactive by in vitro experiments. Here, we show that anle234b has no significant effect on the aggregation process in terms of reducing the $\beta$-structure content. Moreover, we demonstrate that the hydrogen bonding capabilities are autoinhibited due to steric effects imposed by the molecular geometry of anle234b and thereby indirectly confirm the proposed inhibitory mechanism of anle138b. We anticipate that the prominent binding of anle138b to partially disordered and dynamical aggregate structures is a generic basis for anle138b's ability to suppress toxic oligomer formation in a wide range of amyloidogenic peptides and proteins.
\end{abstract}

KEYWORDS: Neurodegenerative diseases, molecular dynamics, MD simulations, small molecule inhibitor, oligomeric aggregate, amyloid

\section{INTRODUCTION}

Providing treatment for neurodegenerative conditions such as Alzheimer's disease $(\mathrm{AD})$ and Parkinson's disease (PD) has been a longstanding quest for fundamental research in academia and pharmaceutical industry as these types of neurological disorders are rapidly becoming a more prevalent cause of death in an aging population. ${ }^{1}$ Currently, no therapies exist that can cure or prevent the irreversible disease pattern in humans and several clinical trials were marked by setbacks in the past. ${ }^{2-4}$

The common defining characteristics in proteopathic neurodegenerative disorders are the misfolding and aggregation of proteins at the molecular level leading to an abnormal accumulation of toxic species and insoluble fibrillar mass in intra- and extracellular space. This process ultimately results in degeneration and death of neuronal cells. ${ }^{5-8}$ At present, the majority of research publications identifies soluble nonfibrillar oligomers as the pivotal agent and primary cause of cytotoxicity for a number of disease-related peptides and proteins. ${ }^{5,6,9-12}$
Inhibition of pathological protein aggregation by targeting diffusible, oligomeric forms and remodeling of toxic conformations by small molecule compounds therefore has been one of many different disease modifying strategies investigated. These treatment strategies aim to reverse, stop or slow down the progression of the diseases in vitro, in cell line models and in preclinical animal studies. ${ }^{13-19}$

Although accounts of biophysical and biochemical properties obtained by direct experimental observation ${ }^{20-24}$ and efforts to determine molecular structures ${ }^{25-28}$ of oligomeric, neuroinflammatory aggregates are accumulating, the picture remains incomplete. The principal structure-neurotoxicity relationship, and whether it is commonly shared by toxic oligomers of different primary sequences, is one of the pressing open questions. Resolving the structures of amyloidogenic peptide

Received: August 24, 2017

Accepted: September 14, 2017

Published: September 14, 2017 

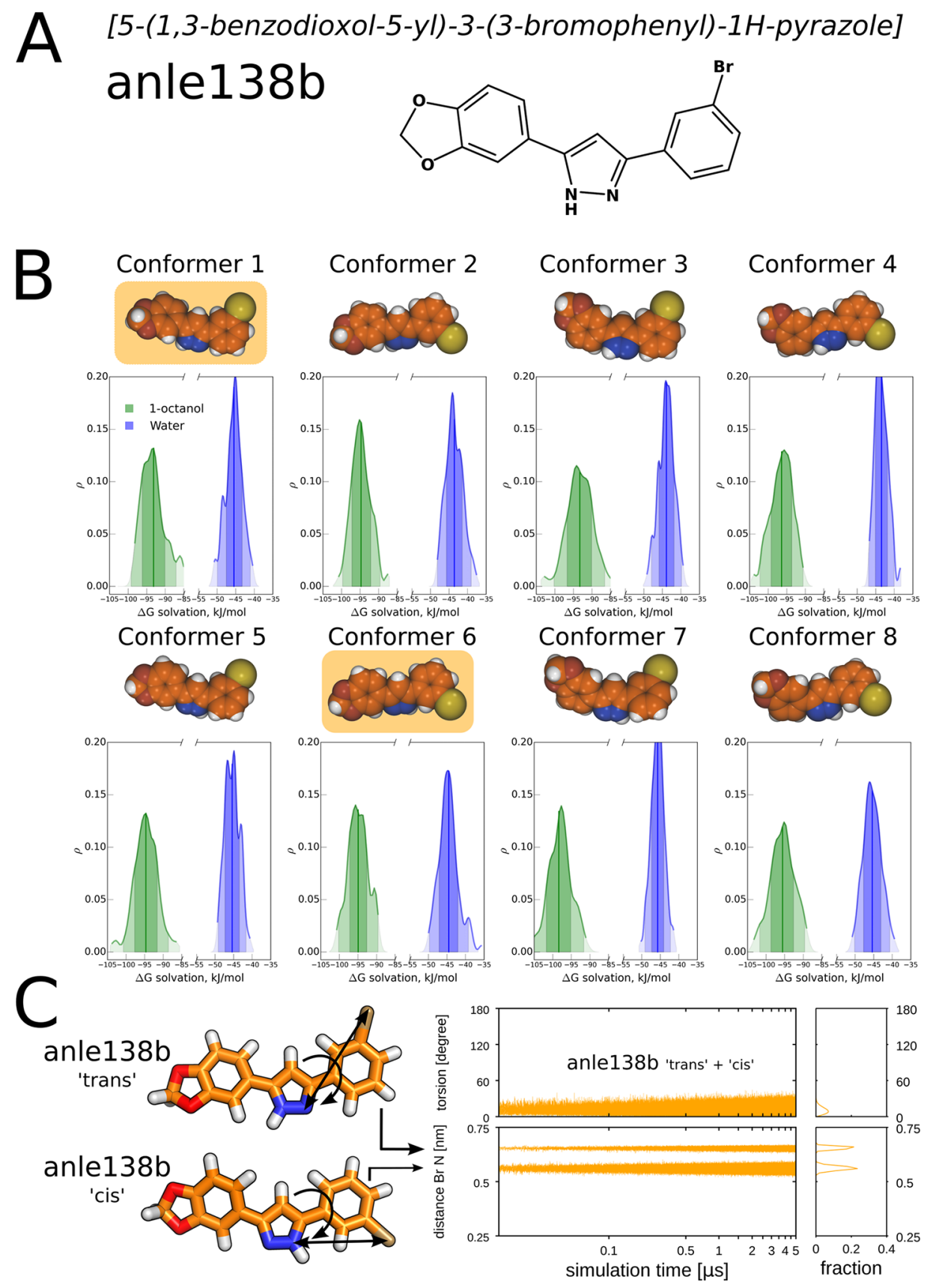

Figure 1. Anle138b conformers and solvation free energies. (A) Chemical structure of anle138b. (B) Principal anle138b conformers, considering all possible different tautomeric forms of the pyrazole moiety and main torsion motifs, are shown together with their associated solvation free energies in water (blue) and 1-octanol (green). (C) Torsion angle distributions of the bromophenylring and distances between bromine and nitrogen atoms of the pyrazole ring collected over all production simulations with anle138b and peptide aggregates at room temperature.

and protein oligomers in solution or in the membrane environment is still a fundamental challenge and proves to be extremely difficult due to the transient and disordered nature of the conformational states populated during the lag phase of amyloid formation. ${ }^{8,29}$ Moreover, essential atomistic details on interaction modes of small molecule inhibitors with the nonfibrillar oligomers are lacking, yet they are of utmost importance for a rational drug development strategy. ${ }^{17,18,30,31}$

Advances of molecular dynamics (MD) simulation methods led to atomic-level insight into the conformational dynamics of disordered proteins and revealed important mechanistic steps of peptide self-aggregation. ${ }^{31-40} \mathrm{MD}$ simulations have also been used to study potent small molecule inhibitors that target oligomeric intermediates and attenuate toxicity, ${ }^{30,35}$ for example, the green tea polyphenol epigallocatechin-3-gallate (EGCG) $)^{19,41-44}$ or the polyolic compound scyllo-inositol. ${ }^{43-46}$ EGCG and scyllo-inositol served as platforms for proof of principle investigations, ${ }^{30}$ giving new insights into molecular mechanisms of small molecule binding affinity and its specificity, ${ }^{47-49}$ as well as structural implications for interactions of inhibitory small molecules with low-molecular weight oligomers. $^{43,48,49}$

Recently, the promising drug candidate anle138b [5-(1,3benzodioxol-5-yl)-3-(3-bromophenyl)-1H-pyrazole] with high central nervous system bioavailability and low toxicity ${ }^{50,51}$ was discovered. ${ }^{51}$ Anle138b was shown to bind peptide aggregates 
in a structure-dependent manner at nanomolar concentrations. $^{51,52}$ In vitro, anle138b suppressed the formation of pathological aggregates and inhibited oligomer formation of the prion protein from multiple tested strains, ${ }^{51}$ the tau protein, ${ }^{53}$ and $\alpha$-synuclein, ${ }^{51,54}$ which is deposited in $\mathrm{PD}$, and other synucleinopathies. It was concluded that the inhibitory effect of anle $138 \mathrm{~b}$ in vitro and in vivo can be attributed to the direct modulation of toxic aggregate species at the oligomer level. $^{51,53,54}$ However, the low water solubility of anle138b (concentrations smaller than $1 \mu \mathrm{M}$ ) precludes a characterization of its interaction with relevant oligomeric aggregation states by high-resolution NMR spectroscopy.

Therefore, we utilize all-atom unbiased $\mathrm{MD}$ simulations to determine how anle138b interacts with small oligomeric aggregates of selected amyloidogenic decapeptides, namely, peptide fragments from human tau, ${ }^{39,55-57} \mathrm{~A} \beta,^{26,58,59}$ human islet amyloid polypeptide (hIAPP), ${ }^{60,61}$ and Sup35p. ${ }^{56,62,63}$ They are experimentally known to be highly aggregation prone and are crucial constituents of $\beta$-structured aggregates formed by their full-length precursor sequences. Our simulation results detailed below provide an in-depth analysis of the principal atomistic interactions that govern anle138b binding and the modulation of peptide aggregate structural ensembles.

\section{RESULTS}

Molecular Geometry and Free Energy Differences of Anle138b conformers. To determine the influence of anle138b on the structure and dynamics of amyloidogenic peptide self-assembly by atomistic MD simulations, we first focused on the conformational preferences of the compound itself (Figure 1A). We investigated the free energy differences between eight principal anle138b conformers, taking into account that the pyrazole moiety of anle $138 \mathrm{~b}$ can exist in two tautomeric forms and considering all main torsion motifs of the bromophenyl and the benzodioxol ring resulting from the two rotatable bonds in the molecule (Figures 1B, S1 and Methods). The solvation free energies for all conformers and tautomers fall within the range of -43 to $-46 \mathrm{~kJ} / \mathrm{mol}$ in water and -93 to $-98 \mathrm{~kJ} / \mathrm{mol}$ in 1-octanol (Figure $1 \mathrm{~B}$, Methods). Figure S1 depicts the results of a separate simulation set (replica exchange simulations in vacuum, see Methods) from which the free energy differences between each conformer per tautomeric form were obtained. It shows that one tautomeric form (conformers 1-4) appears to be strongly preferred in vacuum over the other tautomeric form (conformers 5-8).

From a combination of all the above, we found that (a) conformers 1-4 are more likely to be present in water than 58 and (b) the free energy of conformers $1-4$ is approximately equal. Although the free energies for all four conformers per tautomeric form are very similar and differ only by $1-2 k T$, the initial molecule structure used for parametrization, highlighted in red in Figure S1, affects the simulation outcome to a similar extent of $1-2 k T$.

The analysis over all simulations at room temperature in the present study shows that large torsion angle transitions in anle $138 \mathrm{~b}$ are not sampled during the $\mu$ s simulation time scale, confirming the initial observations of stable, individual anle $138 \mathrm{~b}$ conformers with a planar and rigid molecular geometry (Figure $1 \mathrm{C})$. Because the initially selected anle $138 \mathrm{~b}$ conformers did not interchange with each other, likely due to a high energy barrier associated with them, we selected two representative conformations for the simulations with the peptide aggregates, namely, the anle138b "trans" (conformer 1) and the "cis" (conformer 6), as illustrated in Figures 1B,C and S1. We based our choice on their favorable relative free energy and the fact that these anle138b conformers enfold a diverse set of structural features (tautomeric form of the pyrazole moiety and the torsion space of the bromophenyl ring).

Anle138b Diminishes Interpeptide Backbone Hydrogen Bonds and Alters the Aggregate Size Distribution. Peptide aggregation simulations were started either from randomly dispersed peptide conformations (RNDM setup) or from experimentally determined $\beta$-sheet structures $(\beta$-STR setup) to circumvent possible sampling problems of the aggregated state and to test the impact of anle $138 \mathrm{~b}$ on $\beta$ sheet rich aggregates (Figures $2 \mathrm{~A}, \mathrm{~B}$ and $\mathrm{S} 2$ ). To mimic the

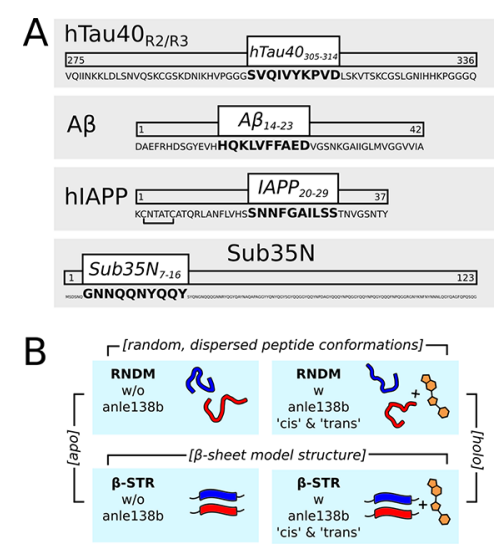

Figure 2. Overview of simulation setup and sequences of simulated peptides. (A) Primary sequence of amyloidogenic decapeptide segments from aggregation prone regions of hTau40, A $\beta$, hIAPP , and Sup35N. (B) Four principal sets of simulations carried out for each sequence and aggregate size (schematic example shown for dimeric simulations).

experimentally confirmed nanomolar solubility of the compound $^{51}$ and to avoid self-association, one anle $138 \mathrm{~b}$ molecule per simulation box was used. Thus, for each of the chosen decapeptide sequences from hTau, A $\beta$, hIAPP, and Sup $35 \mathrm{~N}$, the following sets of simulations per starting structure were run (Figures 2B, S2; Table S1; and Methods): (1) with one anle138b in "cis" conformation and (2) with one anle138b in "trans" conformation (holo simulations), as well as (3) with no anle138b molecule as a control (apo simulations). In total, over 300 multi-microsecond MD trajectories were generated with a cumulative simulation time exceeding $1500 \mu \mathrm{s}$, using an allatom protein and small molecule force field with explicit solvent molecules (see Methods, Table S1). The probability of interpeptide backbone hydrogen bond formation for each of the simulated setups and sequences was analyzed to assess the aggregation extent. The first $2.5 \mu \mathrm{s}$ of each simulation trajectory were neglected to minimize the effect of the respective starting structure.

We found a consistent, prominent reduction in the overall amount of interpeptide backbone-backbone hydrogen bonds in the holo simulations (with anle138b) compared to the apo simulations without the compound (Figure 3). Especially, the holo simulations starting from randomly dispersed peptide conformations (RNDM setup) reveal a substantially higher fraction of peptide chains with few or no intermolecular hydrogen bonds. We also found that the aggregated state is sampled to a smaller extent by 5\% ( $\beta$-STR setup) to up to $25 \%$ (RNDM setup) compared to the apo trajectories, leading to an 

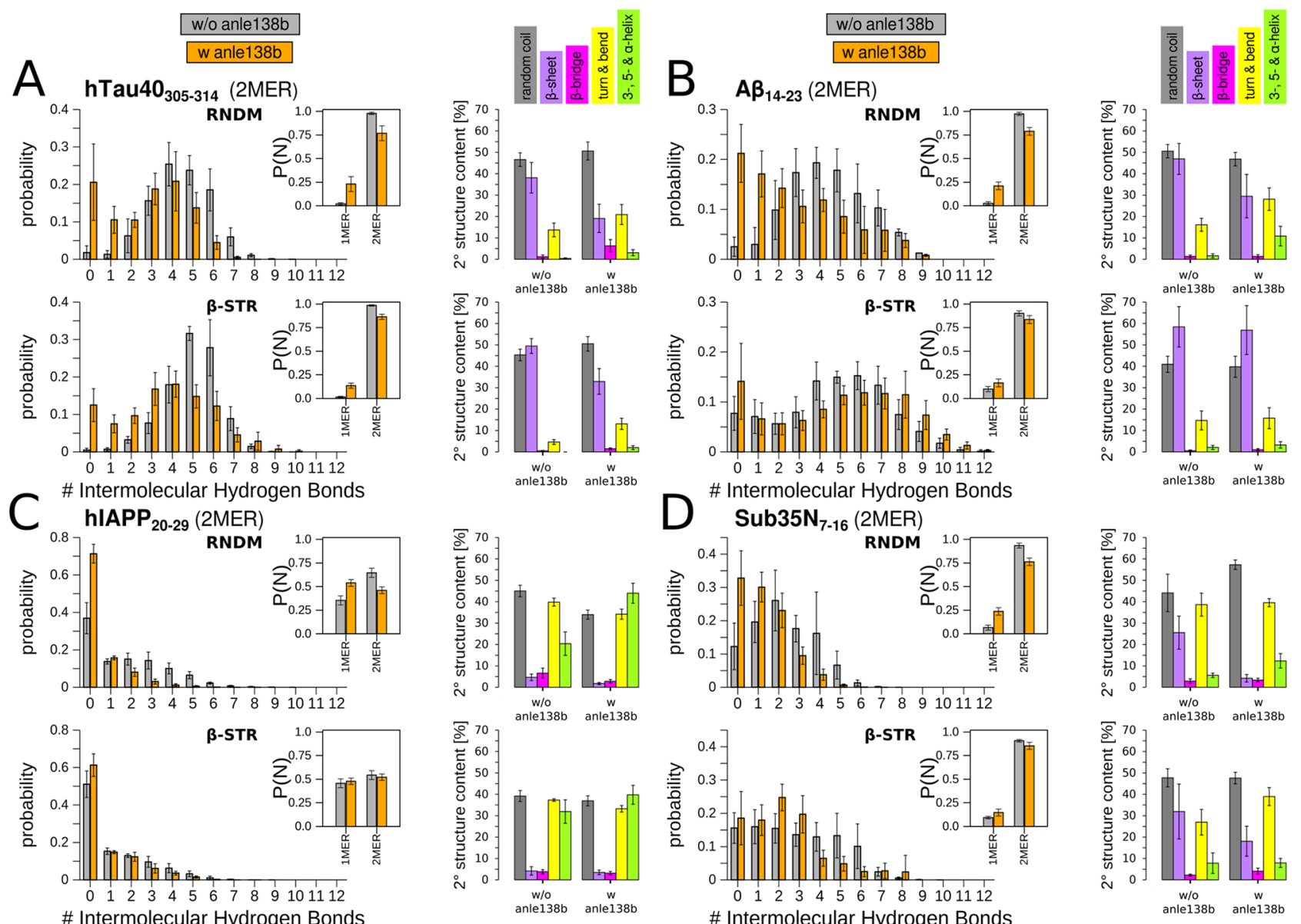

Figure 3. Effect of anle138b on amyloidogenic peptide aggregates. Probabilities of interpeptide backbone hydrogen bonds and the populations of secondary structure elements reported for (A) hTau40 dimer, (B) A $\beta$, (C) hIAPP, and (D) Sup35N simulations with and without anle138b. Top panel shows the results for the RNDM setup, bottom panel shows the results for the $\beta$-STR setup, and insets show the sampled aggregate size distributions. Error bars indicate the standard deviation of the individual simulations from the average calculated over all trajectories.

increased fraction of monomeric peptides. Along these lines, analogous trends are obtained for the dimeric, trimeric and tetrameric hTau 40 aggregates, as well as the dimer simulations of $\mathrm{A} \beta_{14-23}$, hIAPP $22-29$ and the hydrophilic $\operatorname{Sup} 35 \mathrm{~N}_{7-16}$ (Figures 3 and S3).

Anle138b Impedes the Formation of $\beta$-Sheet Rich Structures in hTau40, A $\beta$, hIAPP, and Sup35N Aggregates. On par with the previously presented diminished number of interpeptide backbone hydrogen bonds in the holo simulations, we observe a significant reduction of $\beta$-sheet structure and $\beta$-bridge elements as defined by the DSSP algorithm. ${ }^{64}$ In the apo simulation systems with RNDM setup, $\beta$-strand conformations are the most populated fraction of secondary structure elements, apart from random coil. In the presence of anle138b, the average $\beta$-structure for the hTau $40_{305-314}$ dimer (apo: $38 \pm 7 \%$ /holo: $19 \pm 7 \%$ ), trimer $(37 \pm 9 \% / 30 \pm 4 \%)$, and tetramer $(21 \pm 3 \% / 15 \pm 4 \%)$, as well as for the $\mathrm{A} \beta_{14-23}(40 \pm 6 \% / 25 \pm 9 \%)$ and the Sup $35 \mathrm{~N}_{7-16}(22$ $\pm 7 \% / 4 \pm 2 \%$ ) dimer is decreased, whereas the abundance of turn, coil and helix elements is increased (Figures 3 and S3). The differences in average $\beta$-sheet structure content between apo and holo simulations that started from the $\beta$-STR setup are smaller, but are still significant for the hTau40, A $\beta$ and Sup35N aggregates: hTau40 $305-314$ (apo: $50 \pm 4 \%$ /holo: $33 \pm 6 \%$ ) and Sup35N $\mathrm{N}_{7-16}(27 \pm 11 \% / 16 \pm 6 \%)$ dimers, hTau40 $305-314$ trimer $(42 \pm 4 \%, 40 \pm 2 \%)$ and tetramer $(48 \pm 4 \%, 43 \pm$
$1 \%)$. The average $\beta$-structure content for the $\mathrm{A} \beta_{14-23}$ dimers that started from the $\beta$-STR setup is only marginally different for apo and holo simulations. For the hIAPP ${ }_{22-29}$ dimer, even in the case of simulations starting from initially $\beta$-sheet rich aggregate conformations, only a negligible amount of $\beta$-sheet and $\beta$-bridge content is seen in apo and holo trajectories (Figure $3 \mathrm{C}$ ). The effect of anle138b on the sampling of the $\beta$ sheet rich aggregate states therefore could not rigorously be quantified. Interestingly, in contrast to all other sequences, the hIAPP $_{22-29}$ dimer simulations without anle138b show a high fractional content of helical structures (RNDM setup: $18 \pm$ $5 \% / \beta$-STR setup: $27 \pm 5 \%)$. The high propensity of helix motifs is furthermore increased in the holo simulations of the $\operatorname{hIAPP}_{22-29}$ dimer $(38 \pm 4 \% / 34 \pm 4 \%)$. No significant impact of anle $138 \mathrm{~b}$ is seen on the secondary structure content of the largely unstructured hTau40 $0_{305-314}$ monomer (Figure S4). The holo simulation ensemble, however, shows a moderate increase in the number of intramolecular hydrogen bonds and a higher population of compact structures.

We analyzed the conversions between disordered and ordered oligomeric states for all apo and holo simulations and determined the number of trajectories that sampled ordered aggregates with a $\beta$-strand structure content $>50 \%$. For a statistical error estimate, the Wald method ${ }^{65}$ to determine the $95 \%$ confidence interval was used. The corresponding ratios of ordering transitions are $0.43 \pm 0.21$ ( 9 out of 21 total apo 
trajectories) and $0.25 \pm 0.17$ ( 6 out of 24 holo simulations) for the dimer simulations starting from random, dispersed peptide conformations. The $\beta$-STR setup simulations starting from preassembled, extended $\beta$-sheet structures were first analyzed regarding the transitions from the initially ordered toward disordered oligomeric states with a low $\beta$-structure content $(<20 \%)$. We included an additional set of holo trajectories spawned from stable $\beta$-sheet aggregates that formed during the apo simulations with RNDM setup and are different from the starting structures of the $\beta$-STR setup. Figure 4 shows that the

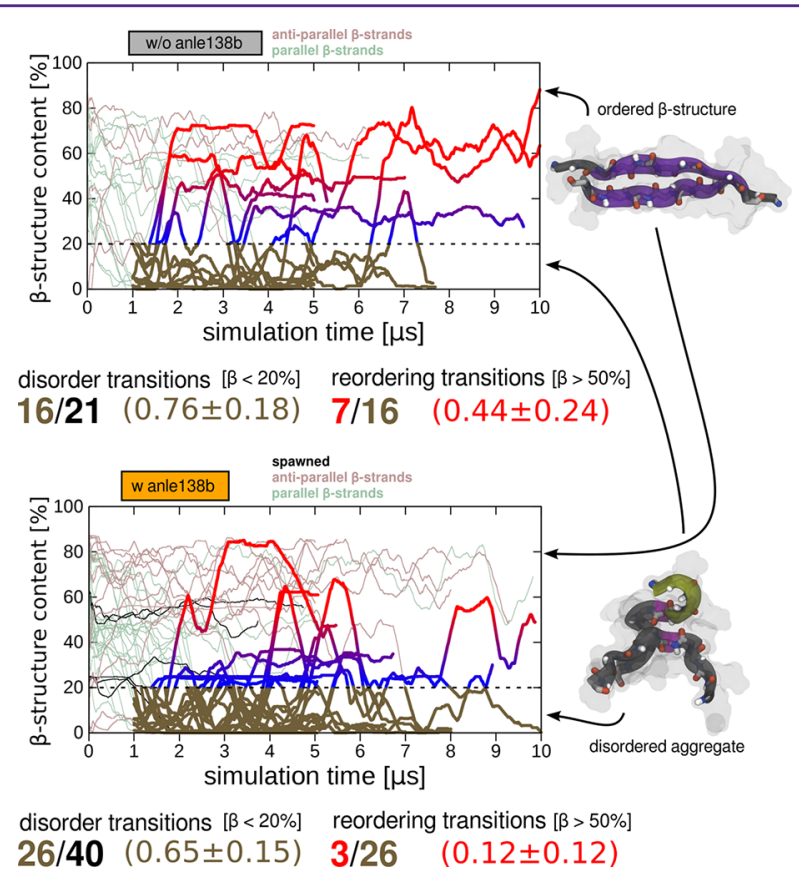

Figure 4. Conversions between ordered and disordered aggregation states. Time dependent $\beta$-structure content shown as 500 ns block average for simulations of hTau40, A $\beta$, hIAPP, and Sub35N dimers started from extended $\beta$-sheet structures with and without anle138b. Disorder transitions of aggregates throughout the trajectories are highlighted by thick lines and brown colors. Red colored lines indicate trajectories with a successful reordering event $(\beta$-structure content $\geq$ 0.5 ) after an initial disordering.

ratio of disordering transitions is very similar for apo $(0.76 \pm$ $0.18)$ and holo $(0.65 \pm 0.15)$ dimer simulations. However, the fraction of trajectories that subsequently returns to aggregate conformations with extensive $\beta$-strand structure is different (apo: $0.44 \pm 0.24$ /holo: $0.12 \pm 0.12$ ). Notably, the individual simulation runs starting from crystallographic and spawned $\beta$ sheet structures with antiparallel strand orientations (dark-red and black colored lines in Figure 4) are more stable than the ones with parallel strand orientations (mint green colored lines in Figure 4). These conformations retain high levels of $\beta$ structure over the course of the multi-microsecond simulation time even in the presence of anle $138 \mathrm{~b}$ and thus contributing to the overall high average $\beta$-structure content of $\beta$-STR setup simulations.

In simulations of hTau40 trimers and tetramers, disordering transitions are less likely to occur (apo: $0.17 \pm 0.15 /$ holo: 0.16 \pm 0.08 ), pointing to a much higher stability of multimeric oligomers already from the trimer onward (Figure S3). The time dependent traces of $\beta$-structure content, the diverging populations of secondary structure elements, and the probability distributions of interpeptide hydrogen bonds with either setup indicate that the full structural equilibration of higher order $\beta$-sheet rich oligomers may lie beyond the time scales accessed in the current study (Figure S3). However, a similar trend in terms of a reduced reordering probability in all trajectories with anle138b is obtained (apo: $1.0 \pm 0$ /holo: 0.36 \pm 0.25 , Figure S3C).

Anle138b Introduces a Population Shift in the Aggregated State Conformational Ensemble. To examine the effect of anle138b in more detail with regard to the conformational ensemble of oligomeric structures and their dominant states, a Principal Component Analysis (PCA, see Methods) was carried out. To compare the conformational variance of oligomeric aggregate conformations in the presence and absence of anle138b, the apo and holo simulation ensembles were projected separately onto the same set of eigenvectors. The eigenvectors were derived by calculating the covariance matrix over all collected frames from the combined sets (RNDM and $\beta$-STR setup) of apo and holo simulations for each peptide sequence. To quantify the sampling differences, similar conformational states according to the following abundant principal aggregate features were merged into "supra-states": antiparallel or parallel, extended $\beta$-strands (ae, pe), partially disordered antiparallel or parallel $\beta$-strands (ad, pd), collapsed and disordered structures (cd), as well as aggregates consisting of intramolecular hairpin structures (hp).

The overall most abundant structure motifs present in the apo ensembles of dimeric hTau 40 and $\mathrm{A} \beta$ aggregates are extended, antiparallel $\beta$-strand conformations with multiple different strand registries. This finding is supported by the corresponding densely sampled states in the PCA projections (regions colored orange and red in Figures 5A and 6A) and the high abundance of ordered $\beta$-strands expressed in terms of the collective variable $\mathrm{CV}_{\beta \text {-strand }} \geq 0.5$ (see Methods, Figures 5B and $6 \mathrm{~B}$ ). A histogram analysis over the "supra-states" of hTau 40 and $\mathrm{A} \beta$ dimer simulations starting from random, dispersed peptide chain conformations further shows a predominantly out-of-register packing for the observed antiparallel $\beta$-strands $\left(a_{\mathrm{OR}}\right.$, Figures $5 \mathrm{C}$ and $6 \mathrm{C}$ ). Sup35N peptide dimers preferentially form partially disordered $\beta$-strands with antiparallel chain alignment (ad) and intramolecular hairpins (hp, Figure S5C). Extended $\beta$-strands, with parallel fibril-like orientations (pe), are the least populated state in all of the apo dimer aggregate ensembles, what is consistent with the low sampling densities of this particular conformation in the PCA projections. Rather, partially disordered parallel $\beta$-structures (pd) are prevalent for the hTau $40, \mathrm{~A} \beta$ and Sup $35 \mathrm{~N}$ dimers. The dimeric state of hIAPP $22-29$ is dominated by collapsed and helical conformations as illustrated in Figure S6A. Ordered $\beta$ structures appear transiently, but none of the dimeric hIAPP $_{22-29}$ conformations are stable beyond the tens of nanoseconds time scale, in contrast to what is observed in the simulation trajectories of the other three amyloidogenic peptide sequences.

PCA projections and representative structures obtained after clustering aggregate conformations reveal that the spontaneously sampled population of conformational states is substantially affected by the presence of anle138b. For the holo ensemble of hTau40 $305-314$ (Figure 5A), A $\beta_{14-23}$ (Figure $6 \mathrm{~A}), \operatorname{Sup}_{35 \mathrm{~N}_{7-16}}$ (Figure S5A), and hIAPP $22-29$ (Figure S6A) dimer structures, we see a broad range of various degenerate minima in the projections and less well-defined major conformational states sampled, compared to the apo ensemble. Judged by the PCA projections, apo and holo simulations cover 


\section{A hTau40 ${ }_{305-314}$ (2MER)}
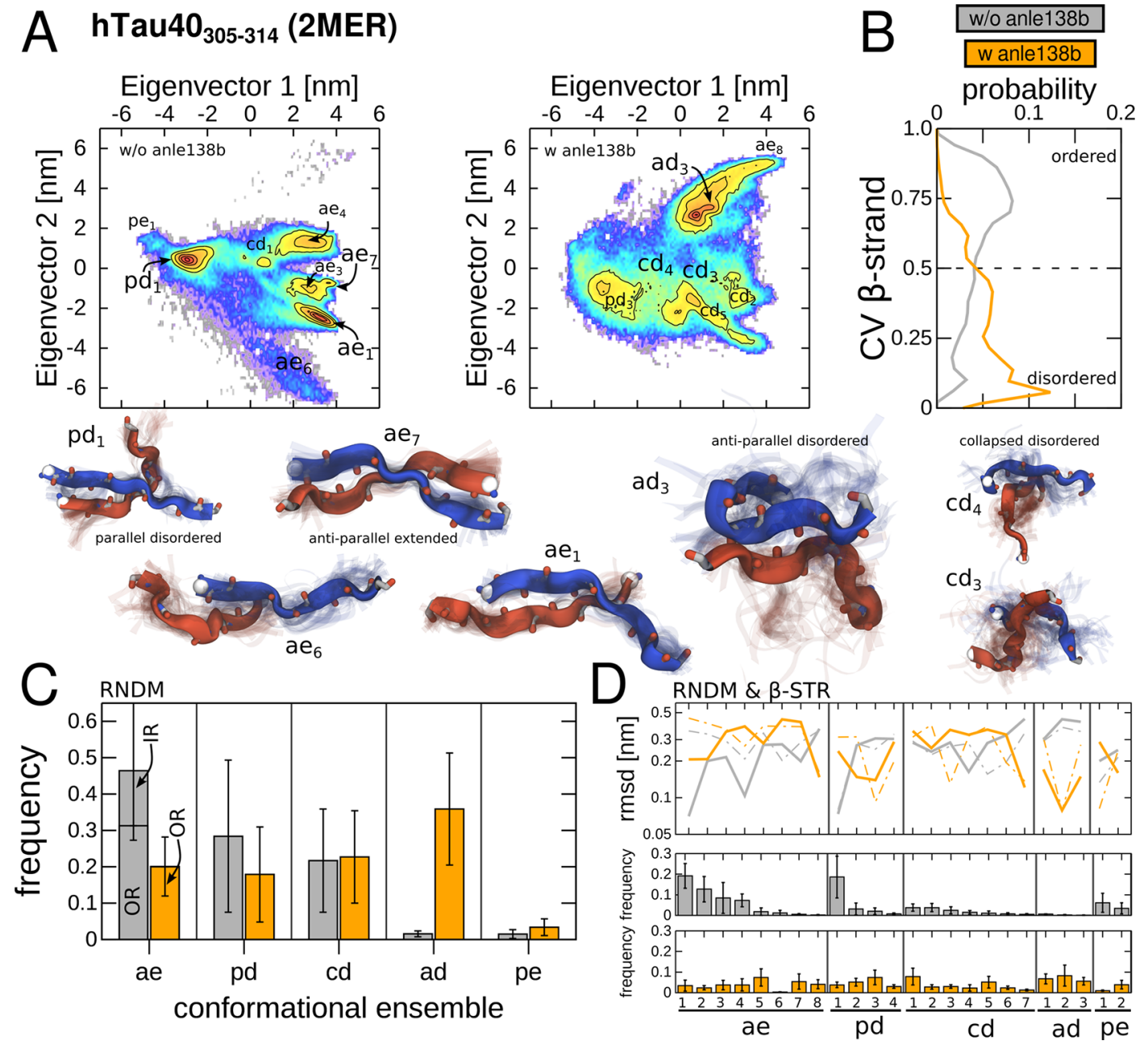

Figure 5. Conformational ensemble of dimeric hTau40 aggregates with and without anle138b. (A) PCA projections of the apo and holo hTau 40 dimer simulations starting from random, dispersed structures are shown together with representative cluster center structures as identified by $\mathrm{k}$ means clustering. (B) Fraction of ordered $\beta$-strand conformations and (C) population frequency of conformational "supra-states". (D) RMSD curves as a function of conformational cluster for two intervals of simulation time (continuous lines $t<2.5 \mu \mathrm{s}$, broken lines $t>2.5 \mu \mathrm{s}$ ) indicate whether the individual substates were sampled during the simulations.

similar regions of the conformational space, but also exist in distinct states not mutually present in apo and holo ensembles. With few exceptions, also the conformational basins common to both the apo and holo structure ensembles differ in their relative sampling density. This difference is indicative of a profound shift in the population of the conformational ensemble induced by anle138b throughout all individual substates, as well as collectively with regard to the lumped "supra-states". In comparison to the apo simulations, the dimeric hTau40 and $\mathrm{A} \beta$ aggregates in the presence of the inhibitor anle138b, ae conformations are depopulated and notably of those structures with out-of-register $\beta$-strands $\left(\mathrm{ae}_{\mathrm{OR}}\right.$ Figures 5C and 6C). Whereas for the Sup35N dimers, especially the intramolecular hairpin conformations (hp) are diminished from the holo ensemble (Figure S5C).

The overall stark depletion of the $\beta$-structured ae and hp conformations raised the question whether they are destabilized by anle $138 \mathrm{~b}$ in a particularly strong manner and rearrange shortly after formation or if their formation is prevented at all over the course of the simulations. Therefore, the sampling of all identified conformational states in the apo and holo ensembles throughout the aggregation process in terms of RMSDs to the individual cluster center structures was analyzed (see Methods). Figures 5D, 6D, S5D, and S6D confirm the suppression of antiparallel $\beta$-strand and hairpin formations in the simulations of spontaneous peptide aggregation with anle138b (RNDM setup). This trend also reflects the overall smaller fractions of fibril-like, extended $\beta$-strand packing in the holo simulations. A concurrent upsurge of collapsed and disordered peptide conformations in the holo aggregate structure ensembles is detected without exception for hTau40, A $\beta$, hIAPP, and Sup35N dimers. The analysis over the individual chain conformations within the larger oligomers, namely the hTau $40_{305-314}$ trimers and tetramers, also shows a higher fraction of disordered versus extended $\beta$-strands, complementing the results of the dimeric state (see Supporting Methods and Figure S7). Similarly to the trends observed for the dimer, an overall higher relative sampling frequency of partially disordered $\beta$-strands and completely unstructured peptide conformations is found in the oligomers simulated in the presence of anle138b (Figure S7). Whereas the populations of extended antiparallel $\beta$-strands in the holo aggregate ensembles are increased and fractions of pe and ae conformations are more balanced between apo and holo ensembles as compared to the dimeric state. 
A $\quad A \beta_{14-23}(2 M E R)$
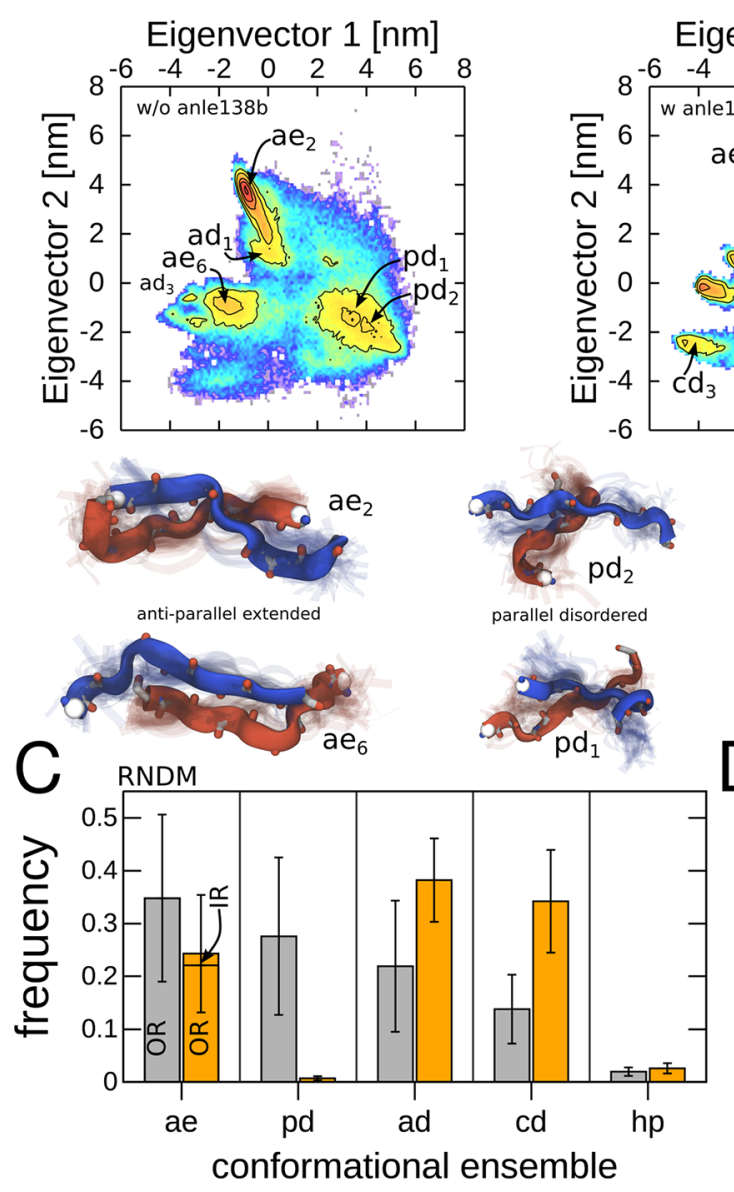

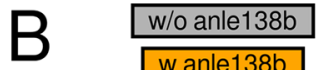

Eigenvector 1 [nm]
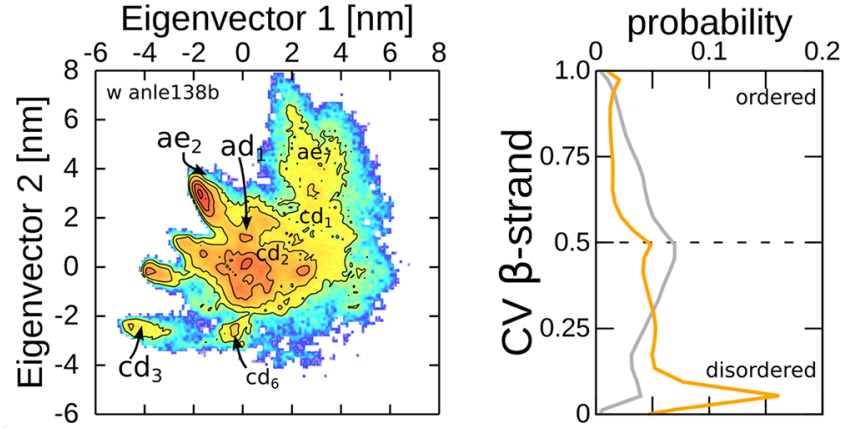
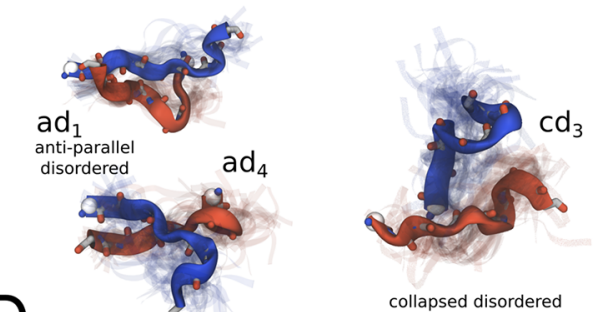

D
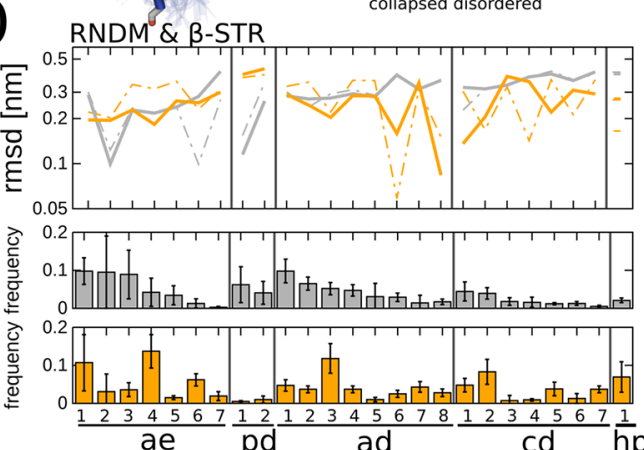

Figure 6. Conformational ensemble of dimeric $\mathrm{A} \beta$ aggregates with and without anle138b. (A) PCA projections of the apo and holo $\mathrm{A} \beta$ dimer simulations starting from random, dispersed structures are shown together with representative cluster center structures as identified by k-means clustering. (B) Fraction of ordered $\beta$-strand conformations and (C) population frequency of conformational "supra-states". (D) RMSD curves as a function of conformational cluster for two intervals of simulation time (continuous lines $t<2.5 \mu \mathrm{s}$, broken lines $t>2.5 \mu \mathrm{s}$ ) indicate whether the individual substates were sampled during the simulations.

Anle138b Shows Specific Interactions with the Peptide Backbone and Multiple Modes of Binding. The contact probabilities of anle $138 \mathrm{~b}$ to peptide main chain and side chain atoms were calculated in simulations of hTau $40_{305-314}$ (Figures 7 and S8), as well as $\mathrm{A} \beta_{14-23}$, hIAPP $_{20-29}$ and Sup35N $\mathrm{N}_{7-16}$ (Figure S9) to better understand the underlying interactions of anle $138 \mathrm{~b}$ with the oligomeric aggregates.

The binding affinity of anle138b to the peptide main chain and side chain heavy atoms is highly independent of the peptide sequence. The residue-based renormalized contact frequencies indicate a preferential binding to the central aromatic and aliphatic residues of the different peptide sequences (Figures 7A, S8A, and S9A): hTau40 $305-314$, Ile308/Tyr310; A $\beta_{14-23}$, Phe19/Phe20; hIAPP $20-29$, Phe23/ Leu27; and Sup $35 \mathrm{~N}_{7-16}$, Tyr13. The renormalized contact probability profile obtained from the $\mathrm{hTau} 40_{305-314}$ simulations displayed in Figure 7A also shows an increased specificity of anle138b binding to the bulky, hydrophobic side chains (Ile308/Tyr310) in the oligomeric aggregates compared to the monomeric state.

The peptide side chain atoms show a rather uniform contact probability across the different parts of anle138b's chemical structure, with the largest number of contacts being made to the central pyrazole ring. With regard to the main peptide chains, the nitrogen atoms of the pyrazole ring consistently feature a significantly larger number of contacts compared to the rest of the compound (Figures 7B, S8B and S9B). The formation of favorable polar contacts between the pyrazole nitrogen sites and the peptide backbone results in prominent hydrogen bonding interactions and explains the larger contact numbers.

The frequency of anle138b hydrogen bonds increases with the oligomer size similarly to the above-described contact probability of anle138b, but hydrogen bonds predominantly form with the peptide backbone motif (Figures 7A and S8A). Interestingly, even sequences with multiple polar side chain groups competing for hydrogen bond formation establish more frequently polar contacts between anle138b and the peptide backbone (i.e., Sup $35 \mathrm{~N}_{7-16}$ and hIAPP $20-29$, Figure S9).

To obtain insight into the anle138b binding mechanism to the ensemble of aggregates, the conformational space of anle138b interacting with peptide oligomers was discretized. The previously described contact patterns of anle138b to oligomeric forms of peptide aggregates in terms of atomic contact numbers and the presence of hydrogen bond 

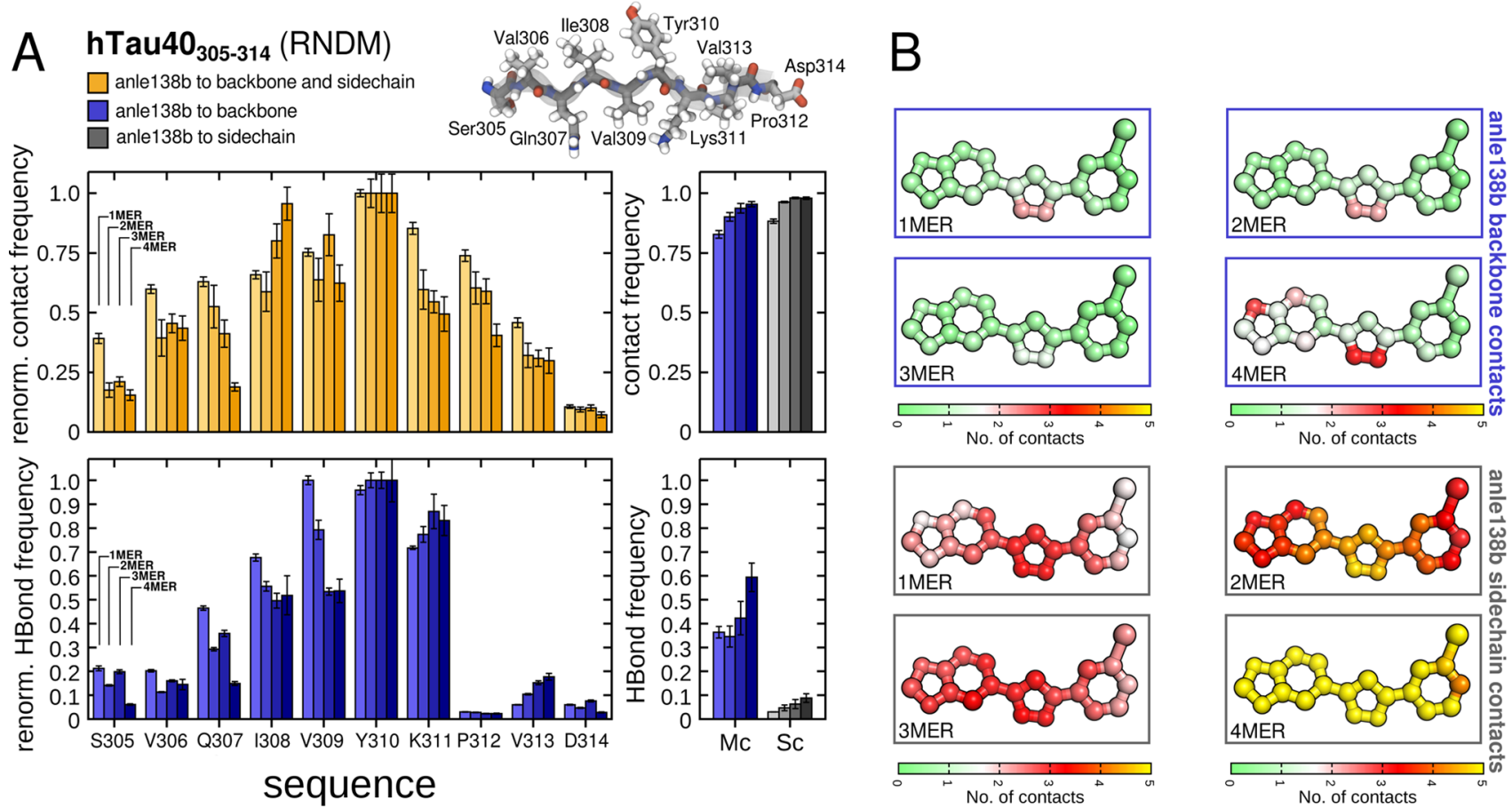

Figure 7. Contact probability and hydrogen bonding interaction of anle138b in hTau40 monomer to tetramer simulations starting from random conformations. (A) Anle138b contact to peptide heavy atoms and hydrogen bond probability are shown as a function of residue index (renormalized) and aggregate size (absolute). (B) Contact frequency of peptide heavy atoms (main and side chain) with anle138b atoms mapped onto chemical structure of the 'trans' conformer.

interactions led us to devise a heuristic classification of five mutually exclusive anle138b binding modes. These modes are detailed in Figure 8A and are defined in the following way: (1) "unbound", no contacts; 2) "partially bound", fewer than half of the anle $138 \mathrm{~b}$ atoms in contact with peptide heavy atoms, mostly describing transient binding events; (3) "docked", half or more of the anle $138 \mathrm{~b}$ atoms in contact with peptide heavy atoms, no hydrogen bond interactions to the backbone atoms; (4) "bound", at least one hydrogen bond between anle138b and peptide backbone or side chain atoms; (5) "blocking", more than $70 \%$ of the anle $138 \mathrm{~b}$ atoms in contact with peptide heavy atoms, at least two hydrogen bonds between anle138b and peptide backbone.

A summary of the mean-first passage time and mean residence time of anle $138 \mathrm{~b}$ in one of the respective binding modes for all simulation systems is shown in Figure S10. The anle138b binding events occur on the hundreds of pico- to nanosecond time scale and anle138b binding with multiple hydrogen bonds takes the longest time to be sampled $(\approx 100$ $\mathrm{ns})$. The lifetime of the overall short-lived binding poses increases with the aggregate size because of the growing number of potentially interacting peptides. Accordingly, the shortest mean residence times of anle $138 \mathrm{~b}$ modes are present in the simulations of the monomeric hTau40 peptide which also show the highest frequency of unbinding events and the largest fraction of unbound or partially bound anle138b (Figure $\mathrm{S} 10$ and $8 \mathrm{~B})$.

The interactions of anle $138 \mathrm{~b}$ with peptide aggregates result in a fast desolvation and burial of the molecule, as illustrated by time dependent anle138b solvation traces from hTau 40 simulations (Figure $8 \mathrm{C}$ ). The number of water molecules in the anle138b hydration shell drops significantly upon further association with the aggregate surface. Nevertheless, the compound maintains a tendency to unbind and exchange with the bulk solvent. We also found a distinct correlation between the desolvation of anle138b's pyrazole ring and the overall loss of solvating water molecules. The number of surrounding waters around anle138b correlates with the abovedescribed binding modes in the following descending order from high to low: partial or transient binding, docked, bound to and blocking the backbone of a peptide chain (Figure $8 \mathrm{C}$ ). The loss of solvation around the small molecule is even more pronounced in simulations of larger hTau40 aggregates (trimer and tetramer) and anle138b was found buried deep within pockets and clefts of the aggregates. Likewise, the insertion and association of anle $138 \mathrm{~b}$ with the oligomeric aggregates results in a substantial reduction of hydrophobic solvent accessible surface area compared to the apo ensembles in all studied peptide aggregate systems.

Moreover, anle $138 \mathrm{~b}$ exhibits a structure dependent binding as the differing binding mode probabilities for RNDM and $\beta$ STR simulation systems summarized in Figure $8 \mathrm{~B}$ show. In fact, all preassembled $\beta$-sheet models that show a high structural stability throughout the course of the simulations (hTau $40_{305-314}$ dimer to tetramer and $\mathrm{A} \beta$ dimer) are found with a lower abundance along with a slower mean-first passage time of anle138b binding modes involving peptide backbone hydrogen bond interactions (Figure S10).

To investigate the binding of anle $138 \mathrm{~b}$ to amyloid cross- $\beta$, fibril-like structures (as opposed to metastable, nonfibrillar oligomers described so far), simulations of tetra- and octameric $\beta$-sheet model structures with positional restraints imposed on their backbone atoms were set up (Figure S11).

Simulations of these highly ordered, fibril-like structural arrangements yield an overall higher fraction of "docked" anle138b molecules compared to the simulations where the 
A
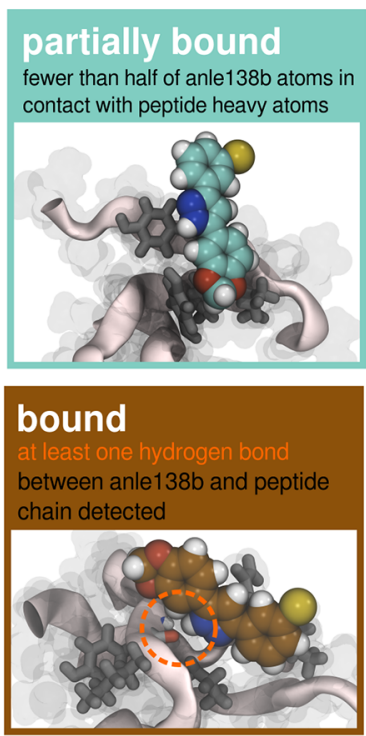

unbound
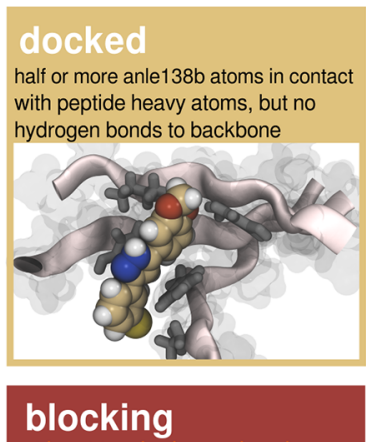

C
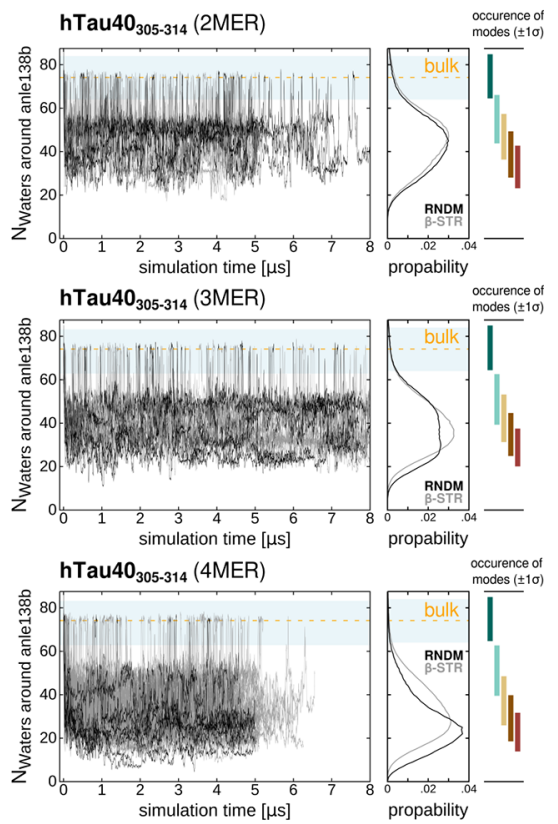

B
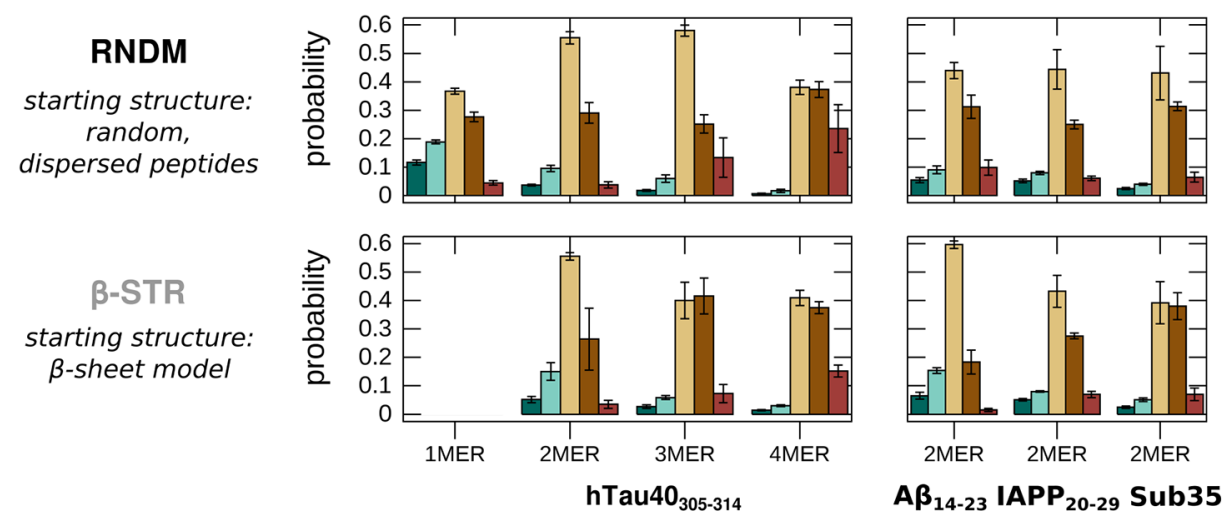

$A \beta_{14-23}$ IAPP $_{20-29}$ Sub35N $_{7-16}$

Figure 8. Characterization and probability of anle138b binding modes. (A) Representative simulation structures and characterization of main anle138b binding modes. (B) Population of binding modes as a function of the simulation system (aggregate size, starting peptide conformation, sequence). (C) Number of hydration water molecules around the anle $138 \mathrm{~b}$ molecule as a function of simulation time (hTau $40_{305-314}$, monomer to tetramer). Light blue areas illustrate the fluctuation of solvent molecules around the anle138b compound in the bulk without any peptides present. Color bars show the relation of small molecule hydration and occurrence of a certain binding mode (bar length $= \pm 1 \sigma$ ).

peptide chains were free to rearrange as shown by the binding mode probability (Figures S11 and $8 \mathrm{~B}$ ). The "docked" binding mode is almost exclusively found in simulations of nonfibrillar hTau40 structure models based on PDB entry 4E0M with antiparallel strands, featuring an extended hydrophobic patch on one side of the $\beta$-sheet to which anle $138 \mathrm{~b}$ binds in an isotropic fashion. In contrast, anle $138 \mathrm{~b}$ binds to all fibrillar structures with single or double layer parallel in-register $\beta$ strands in much more defined poses. The representative structures in Figure S11 show the molecule being inserted into the hydrophobic side chain grooves and aligned parallel to the fibril axis. Anle138b binds to single and double layer $\beta$-sheets of the same principal cross- $\beta$ structure in a different fashion; namely, "docked" and "partially bound" modes and virtually no hydrogen bonding to the peptide backbone in case of the unpaired single layer $\beta$-sheets occur. Simulations of paired $\beta$ sheet layers with a steric zipper type interface show an increased fraction of 'bound' and 'blocking' modes. Here, anle $138 \mathrm{~b}$ occludes and caps the respective solvent-exposed tips of the fibrillar structure.

The Inhibitory Effect of Anle138b Is Directed toward the Disordered Structure Ensemble. To examine structure dependent binding characteristics of anle138b, we introduced an enrichment factor (EF) analysis. The EF of the individual binding modes was computed for the aggregate conformations as a function of $\beta$-sheet content intervals as follows:

$$
\begin{aligned}
\mathrm{EF}_{\text {mode }}^{\text {interval }}= & \frac{\text { no. of mode structures per interval }}{\begin{array}{l}
\text { no. of total mode structures } \\
\text { total no. of peptide conformations }
\end{array}} \\
& \times \frac{\text { no. of peptide conformations per interval }}{\text { nof }}
\end{aligned}
$$

An $\mathrm{EF}_{\text {mode }}^{\text {interval }}$ value larger than 1 indicates that a given anle $138 \mathrm{~b}$ mode at the defined $\beta$-sheet interval is found more frequently 
A
B
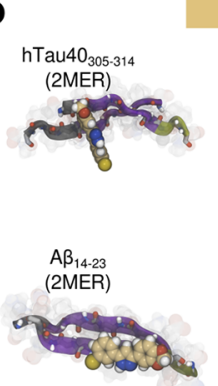

hIAPP $_{20-29}$
(2MER)

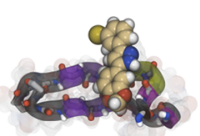

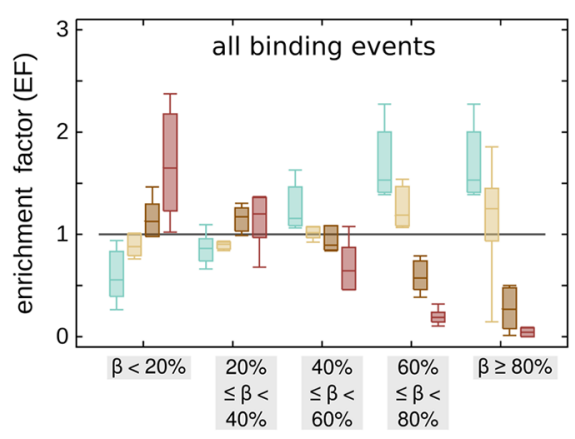

$0 \%$
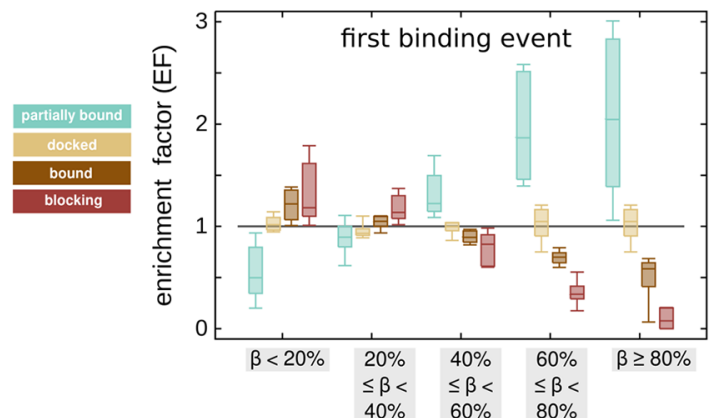

bound

$\begin{array}{lll}\leq \beta< & \leq \beta< & \leq \beta< \\ 40 \% & 60 \% & 80 \%\end{array}$

blocking

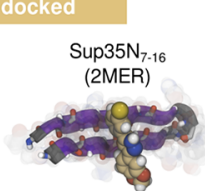

hTau $40_{305-314}$ (3MER)

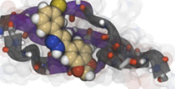

hTau $40_{305-314}$ (4MER)
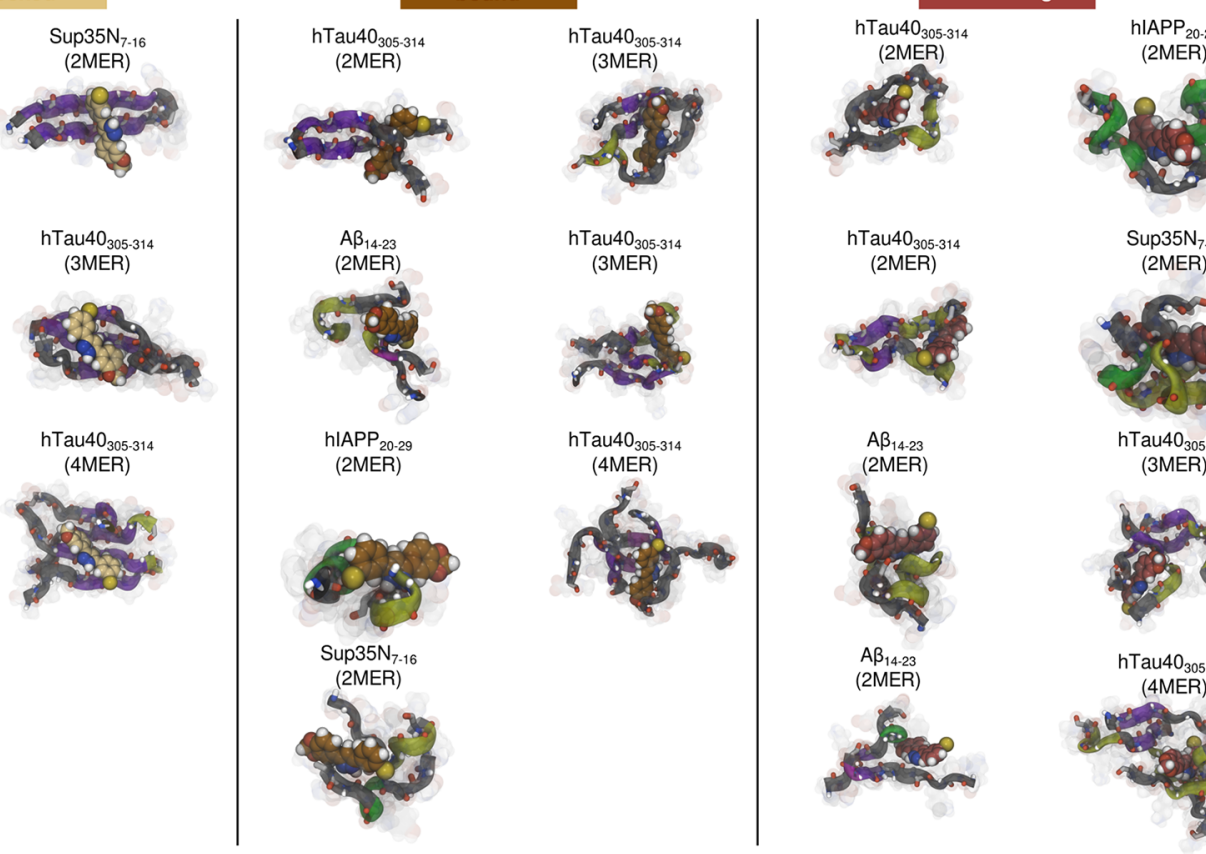

hIAPP $20-29$
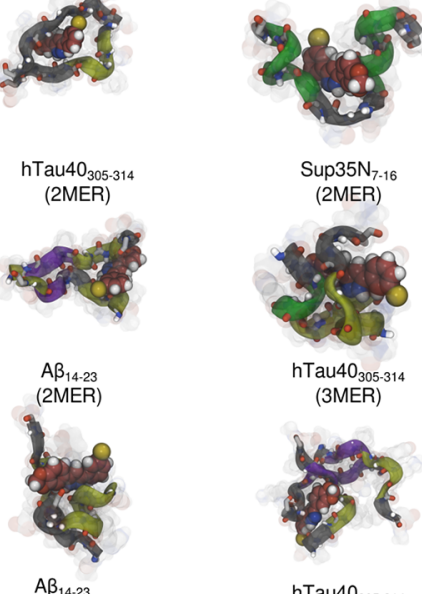

(3MER) (2MER)
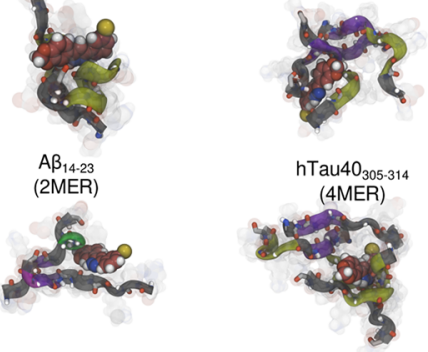

Figure 9. Structure dependent binding characteristics of anle138b. (A) The enrichment factor of anle138b binding events per mode as a function of $\beta$-structure content is shown as cumulative distributions for all simulated sequences and aggregate sizes in box plots. Box and whisker indicate $1 \sigma$ and $2 \sigma$ of the distribution widths, respectively. (B) Representative structures of peptide aggregates with anle138b in docked, bound, and blocking modes.

than the ensemble average. Figure $9 \mathrm{~A}$ shows the binding mode populations across the different conformational states in the aggregate structure ensembles. Anle138b's first as well as the total number of binding events involving the "bound" and "blocking" mode occur overall preferentially to oligomer conformations of the disordered and collapsed structure ensemble ( $\beta$-structure content below 20\%). The EF, i.e., the relative probability for the "bound" and "blocking" modes is the smallest for the $\beta$-sheet rich, ordered oligomeric states $(\beta$ structure content above $60 \%$ ). We thus found a preferential occurrence of anle138b with hydrogen bonding interactions ("bound" and "blocking" modes) to aggregate structures that exhibit a low $\beta$-structure content and small fraction of extended fibril-like $\beta$-strand conformations (Figure 9A). The outcome of the EF analysis is invariant across sequence space and the probed aggregate size despite the lower compound-to-peptide ratio in trimer and tetramer simulations.

As illustrated by the representative structures in Figure 9B, the rigid, planar geometry and hydrogen bonding capability of the small molecule often results in the partial or complete wedging between aggregate chains, leading to the break up of intermolecular peptide backbone interactions and to the disruption of ordered $\beta$-strand arrays. One can further assess that oligomers bound to anle $138 \mathrm{~b}$ via the previously characterized "bound" and "blocking" modes adopt wrapped up, coiled and collapsed structures. In contrast, extended $\beta$ structured oligomers (if sampled) preferentially feature "docked" and "transient" anle138b binding modes. In these instances, the compound is often well aligned with the $\beta$ strands and attaches to the molecular surface of peptide side chain groups rather than to the peptide backbone.

Control Experiment with Inactive Anle234b Verifies Anle138b's Mode of Action. To validate the hypothesized anle $138 \mathrm{~b}$ mode of action, we simulated the inactive compound anle $234 b^{51}$ together with the hTau40 dimer system. Anle234b is a structural isomer of anle $138 \mathrm{~b}$ and the two molecules differ only in the position of the bromine substitution on the phenyl ring (ortho- for anle234b instead of meta- for anle138b). In contrast to the anle $138 \mathrm{~b}$ simulations, anle $234 \mathrm{~b}$ undergoes torsional motion of the bromophenyl ring, resulting in a stable conformation with the bromine atom in ortho-position in close proximity to the nitrogen atoms of the pyrazole moiety (Figure 10A).

The steric hindrance imposed by the bulky bromine group leads to a "torsional inactivation" of anle234b impeding the interactions of the bromophenyl and the pyrazole ring with the peptide backbone. The significantly reduced contact probability of anle $234 \mathrm{~b}$ to the peptide backbone atoms leads to a reduced 


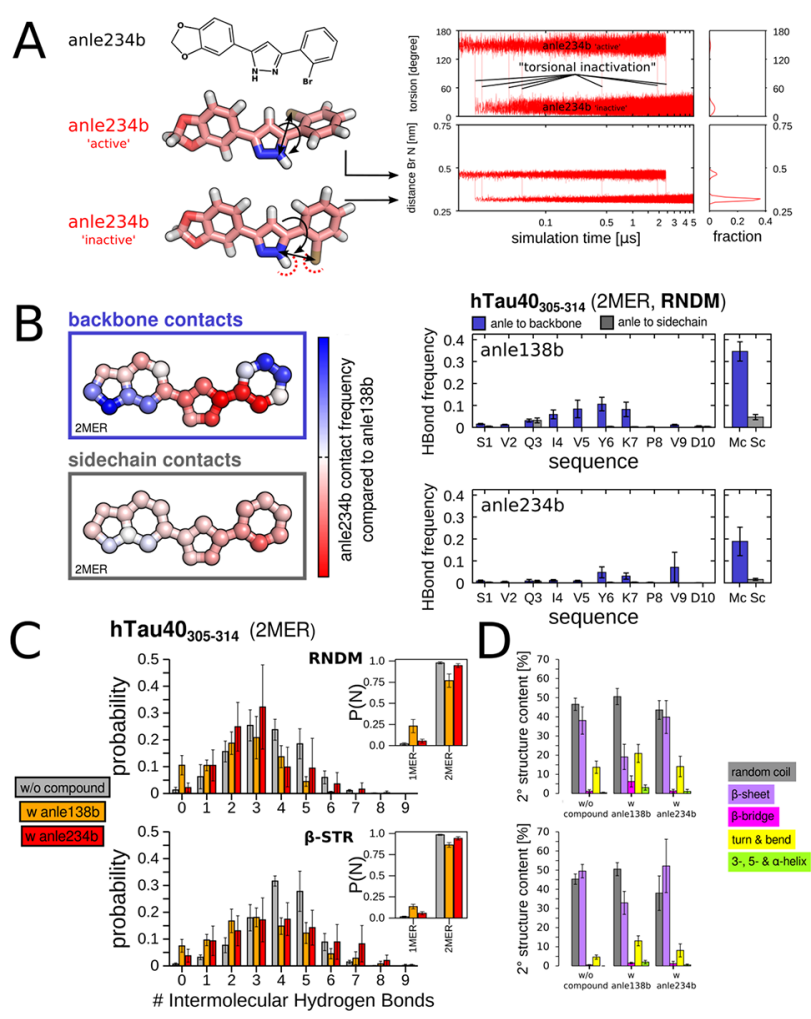

Figure 10. Inactive compound anle $234 \mathrm{~b}$ does not affect aggregation of hTau40 peptides. (A) Anle234b conformations over all hTau40 dimer simulations carried out are shown as torsional distributions of bromophenyl ring and distances between bromine and nitrogen atoms of pyrazole moiety. (B) Contact frequency of anle138b/234b to peptide heavy atoms mapped onto the chemical structure and comparison of anle138b and anle234b hydrogen bond probability as a function of peptide residue index. (C) Intermolecular main chain hydrogen bond and aggregate size distributions, as well as (D) ensemble-averaged populations of secondary structure elements are shown for simulations without as well as with the anle138b and anle234b compounds.

hydrogen bonding capability compared to anle138b, whereas the side chain contact probability based on the analysis of nonspecific and mainly hydrophobic contacts is very similar for both investigated molecules (Figure 10B). Interestingly, the average $\beta$-structure content levels of apo and anle $234 \mathrm{~b}$ simulations are comparable and are both significantly higher compared to the respective simulations with anle138b (Figure 10C). We also found a larger number of interpeptide backbone hydrogen bonds and a larger fraction of dimeric peptide chains compared to the anle138b simulations (anle138b/anle234b, RNDM setup: $77 \pm 8 \% / 95 \pm 2 \%, \beta$-STR setup: $87 \pm 3 \% / 94 \pm$ $2 \%)$, suggesting little to no potency of anle $234 \mathrm{~b}$ to inhibit $\beta$ oligomer formation in the simulations.

\section{DISCUSSION}

Despite considerable progress in uncovering the etiology of pathogenic aggregates in amyloid diseases, the molecular footprints underlying their formation and potential inhibition mechanisms remain largely in the dark. ${ }^{15}$ Anle $138 \mathrm{~b}$ is a newly discovered synthetic compound which has emerged as a potent inhibitor of toxic oligomer formation. ${ }^{51,53,54}$ Yet, structural information regarding anle138b's conformational preferences and its specific interactions with self-assembled amyloid aggregates is limited. The main goal of the present study was therefore to understand the mode of action and the impact of anle $138 \mathrm{~b}$ on the conformational changes during the primary nucleation steps of small oligomeric aggregates formed by fragments of hTau, A $\beta$, hIAPP, and Sup35 prion protein.

As observed in our all-atom MD simulations, the small aromatic molecule anle $138 b^{50,51,53}$ is a planar system without rotations around the bonds connecting the central pyrazole ring with the 3- and 5-aryl moieties. Previous gas phase IR experiments $^{66}$ and solution-state NMR spectroscopy ${ }^{67}$ investigating the smaller 3-methyl-pyrazole and a class of aminopyrazole analogues $^{68,69}$ in complex with model peptides demonstrated that the pyrazole heterocycle is able to form bidentate hydrogen bond contacts to the carbonyl oxygens and amide hydrogens of the peptide backbone. Similarly, we found that the pyrazole of anle138b prevalently established transient intermolecular hydrogen bonds to the peptide backbone. The importance of the pyrazole motif for the high activity of the compound is illustrated by variations to the molecular structure as found for other members of the focused diphenyl-pyrazole compound library; anle138b was eventually selected from ref 51. Replacing the pyrazole core group of anle $138 \mathrm{~b}$ with imidazole (sery345) or isoxazole (sery338), thereby altering the hydrogen bonding characteristics, was found to diminish the antiaggregation effect of these compounds. ${ }^{51}$ Remarkably, it was previously shown that also a minor change in the bromine substitution pattern on the 5-phenyl ring from meta(anle138b) to ortho-position (anle234b) renders the diphenyl-pyrazole compound inactive. ${ }^{51}$ Our analysis reveals that the rotation of the bromophenyl ring in anle $234 \mathrm{~b}$ is less restricted compared to anle138b and results in a stable conformer that imposes steric hindrance on the pyrazole ring. Therefore, anle234b is apparently not able to recognize the peptide backbone to the same extent as anle138b.

Anle138b has been shown to reduce the abundance of neuropathological oligomers formed by the prion protein, ${ }^{51}$, the tau protein, ${ }^{53}$ and $\alpha$-synuclein. ${ }^{51,54}$ On the molecular level, our simulations support the notion that the efficacy of anle $138 \mathrm{~b}$ is neither specific to the sequence nor a certain amino acid composition due to the high affinity of anle138b toward the misfolded states of the aggregation-prone proteins, therefore suggesting a universal inhibition effect against any amyloidogenic sequence. Our simulations show that anle $138 \mathrm{~b}$ readily attaches to hydrophobic parts of the oligomeric aggregate surface and especially the aromatic residues, as universally present in the investigated sequences. The present data on solvation free energies moreover demonstrate the strong preference of anle138b for apolar interactions, contributing to the stable association of the compound to the peptides, as well as the successful competition with peptide-peptide selfassembly. ${ }^{19,70}$ As anle 138 b reversibly buries itself into the oligomeric aggregate structures, hydrogen bonding interactions between the compound and the peptide backbone are reinforced. Interestingly, a synergistic effect of hydrogen bonds and hydrophobic contacts is also reported for previously designed aminopyrazole $\beta$-sheet ligands, ${ }^{69,71}$ which share structural resemblance to anle $138 \mathrm{~b}$ investigated here. Along these lines, the fraction of anle138b molecules in our simulations with direct interactions to the peptide backbone is found to be the smallest for monomeric peptides and increases with oligomer size, i.e., the extent of potentially exposed hydrophobic groups. These observations are consistent with previous experimental evidence from in vitro fluorescence spectroscopy of anle138b indicating high affinity binding to 
aggregates of $\alpha$-synuclein and no specific interactions to the monomeric form. ${ }^{52}$ In addition, anle $138 \mathrm{~b}$ was found to be shielded from the surrounding water molecules despite the fact that a particular tight binding to oligomeric forms was not observed. $^{52}$

It is worth noting that the observed altering effect of anle $138 \mathrm{~b}$ on the structural transitions and population of the conformational ensembles in our simulations is present even at the high $10 \mathrm{mM}$ peptide concentration, which strongly favors aggregation through the frequent encounter of the peptide molecules. It is reasonable to assume that the inhibitory effect is also affected by the compound-to-peptide-chain molar ratio. The number was fixed to one anle $138 \mathrm{~b}$ molecule per simulation box throughout the present study and resulted in a lower effective compound concentration for the higher order oligomeric states. We therefore expect the lower effective concentration of anle $138 \mathrm{~b}$ to explain the less significant differences between the apo and holo ensemble of the trimeric and tetrameric aggregates in terms of secondary structure and sampling of conformational states compared to the dimeric state.

The simulation ensembles reported here show dominant oligomer conformations that are consistent with the crystal structures of short model peptides ${ }^{26,72-74}$ and findings from FTIR measurements of full-length systems, ${ }^{21,24,60}$ concerning the following principal features: (a) Observation of multiple distinct strand-to-strand and sheet-to-sheet packings that are different from the mainly parallel in-register $\beta$-sheet of the fibrillar state; ${ }^{72,75}$ (b) the oligomeric species are partially structured and less compact compared to the highly organized amyloid fibrils; ${ }^{21,75}$ (c) structural studies of several amyloidogenic sequences; such as the $\mathrm{A} \beta$ peptide, ${ }^{26,58,73,75,76}$ tau, $^{72}$ IAPP,,$^{74,77}$ or $\alpha$-synuclein; ${ }^{21,78}$ report antiparallel $\beta$-sheet conformations as a prominent hallmark of early oligomeric aggregates; $^{11}$ (d) the prevalently observed out-of-register $\beta$ sheets are a common structural motif in amyloid aggregates. $^{27,58,73,74,77}$

Over the course of our multi-microsecond simulations, we observed partially stable oligomers repeatedly transitioning between disordered aggregation states and ordered structures with a high fraction of antiparallel $\beta$-sheet motifs. These results are in line with previous atomistic simulations of peptide dimers $^{32,35,39,47}$ and higher-order assemblies ${ }^{33-35,79,80}$ that predict amyloid-like peptide aggregate structures with antiparallel $\beta$-sheet content to be stable also in a noncrystalline environment. In addition, computational ${ }^{33-35,81-83}$ and experimental $^{20,22}$ work characterizes prenucleated, small oligomers as metastable due to the small free energy barriers between the different conformational states. A kinetic control of the self-assembly reaction therefore could give rise to early aggregate intermediates rich in antiparallel $\beta$-strand structure. $20,22,34,82,83$

For the present work, we choose the AMBER99SB*$\mathrm{ILDN}^{84,85}$ force field with solvent molecules explicitly represented by the TIP3P water model ${ }^{86}$ for all simulations. This modified version of AMBER99SB ${ }^{87}$ was optimized toward a balanced sampling of secondary structure elements and shows improved performance in folding simulations and better agreement with experimental data compared to older generations of force fields. ${ }^{88}$ In a previous study, ${ }^{34}$ we critically assessed the accuracy with which nonpolarizable MD force fields can capture the heterogeneous conformational states of peptide aggregation. Apart from the required extensive sampling, the comparison between different state-of-the-art force field variants revealed qualitative agreement between the structural properties of the aggregates and the global features of the self-assembly process. The AMBER99SB*-ILDN force field was shown to be sufficiently accurate to distinguish between aggregation-prone and nonaggregating sequences, without a particular bias toward the aggregated state and able to sample diverse conformations consistent with experimental structures of peptide aggregates as also demonstrated by others. ${ }^{89}$

So far, the link between the cytotoxic and the specific conformational properties of oligomeric aggregates has not been unequivocally established and the putative distribution of soluble species that mediate synaptic toxicity during amyloidogenic aggregation reportedly involves structures as small as dimers, trimers, but also larger multimers. ${ }^{15,19,20,22,89}$ However, in recent years, several studies assert that the $\beta$-sheet geometry makeup of toxic oligomers is distinct from the cross- $\beta$ structure of amyloid fibrils. $9,20,21,27,75,76$ Collectively, a variety of techniques with high structural resolution revealed out-ofregister antiparallel $\beta$-strands packed together in cylindri$\mathrm{cal}^{21,25,27}$ twisted, $^{89}$ or paired arrays, ${ }^{72,73}$ as well as oligomers with larger barrel-like topology ${ }^{28,90}$ or quaternary structures composed of nested and sheared $\beta$-hairpins. ${ }^{27,76}$ In addition, the recognition of nonfibrillar and toxic species by conformation sensitive antibodies (A11) ${ }^{9,91}$ suggests a generic molecular basis eventually shared by several different amyloid proteins independent of primary sequence. ${ }^{9,63}$ Therefore, these models could serve as a template for amyloid pores or channellike structures commonly associated with the lipid membrane disruption and oligomer-induced toxicity. $5,9,27,54,89$

The present results show that substoichiometric concentrations of anle $138 \mathrm{~b}$ considerably hindered the spontaneous formation of ordered oligomers with an extended $\beta$-structure, although a complete prevention of aggregation was not found. Rather, a profound population shift in the oligomeric structure ensemble was observed, suggesting that early intermediates along the oligomerization pathway will be arrested in disordered and collapsed aggregation states or incapacitated to convert to $\beta$-sheet rich conformations once direct interaction with anle $138 \mathrm{~b}$ occurs. In addition, the sampling of antiparallel and in particular out-of-register $\beta$-strand conformations compatible with the structure of potentially toxic oligomers was inhibited compared to the simulations without compound. Anle138b did not directly divert oligomers with highly ordered, stable $\beta$-strands toward disordered conformations, however, once they transiently exchanged with the disordered structure ensemble, a return to ordered $\beta$-sheet rich conformations was impeded by anle $138 \mathrm{~b}$ in a majority of the simulations. The examined binding dynamics clearly show that interactions with peptide backbones in partially disordered and nonfibrillar oligomers were longer lived and faster accessible for anle138b. The compound was found to prominently wedge in between the peptide chains of these fluctuating aggregate structures with low nematic order, but without assuming a single well-defined binding pose. As a result of direct peptide-pyrazole interactions, a loss in interpeptide hydrogen bonds was observed in the simulations. Interestingly, this binding mode is different from the complementarity binding and capping of ordered $\beta$-sheet structures reported for $\beta$-sheet ligands ${ }^{68,69,71}$ and peptide based $\beta$-sheet breaker constructs. ${ }^{30,72,92-94}$ In contrast to all the other investigated sequences, the hIAPP simulations revealed a predominantly $\alpha$-helical dimer. Our findings, however, are in agreement with experimental evidence and a previously 
proposed mechanism suggesting a helical intermediate onpathway to fibril formation. ${ }^{95}$ Moreover, the hIAPP simulations with anle138b demonstrated the ability of the compound to also destabilize oligomeric aggregates with mainly helical secondary structure by perturbing the balance between interand intramolecular hydrogen bonding.

Finally, the aforementioned converging lines of experimental evidence substantiate that low order oligomeric peptide species account for cytotoxic effects in several devastating neurodegenerative diseases. ${ }^{6,7,9,10,12,23,63}$ However, that does not exclude the relevance of other types of toxic aggregates, ${ }^{22,74,77}$ in part due to the highly polymorphic nature of amyloid intermediates and their aggregation end-states. ${ }^{7,8}$ The presence of multiple diverse and interdependent aggregation states in turn may also suggest that small molecule inhibitors interact in different ways with them. ${ }^{48,96}$ Our simulations of larger and highly ordered $\beta$-sheet filament arrangements show an increased burial of anle138b in side chain grooves and cavities at the expense of anle138b conformations with direct polar interactions to peptide backbones, in contrast to the frequent occurrence of such binding modes in the simulations of the partially disordered and nonfibrillar oligomers. Moreover, we found that anle $138 \mathrm{~b}$ hydrogen bond interactions to the cross- $\beta$ steric zipper motif are preferred over those with extended open edge $\beta$-strands of unpaired, single $\beta$-sheet filaments. These findings prompt a number of questions regarding a potential multimodal interaction mechanism of anle $138 \mathrm{~b}$ that warrant further investigation into the specifics of the aggregation kinetics: ${ }^{96}$ Do fibril tips and open edge strands of double layer $\beta$-sheets constitute a viable interaction site for anle $138 \mathrm{~b}$ as well? Is anle $138 \mathrm{~b}$ therefore potentially able to interfere with fibril growth and elongation? Does anle138b bind to the fibril surface such that secondary nucleation, which is a proposed important pathway for the catalysis and release of toxic oligomeric species, ${ }^{96,97}$ is suppressed? Does anle138b prevent fibril fragmentation as proposed for compounds such as polythiophenes ${ }^{98}$ and orange $\mathrm{G}^{99}$ that bind and apparently stabilize fibrils and ordered protein aggregates?

\section{CONCLUSION}

We studied the molecular mode of action for the small molecule inhibitor anle $138 \mathrm{~b}$ by means of all-atom MD simulations. Our work provides insight into the previously unknown anle138b binding mechanism to small oligomers and its ability to directly modulate these structures during the process of peptide aggregate formation. In summary, we conclude the following about the molecular events that underlie the $\beta$-sheet inhibition mechanism of anle138b: The binding characteristics of anle $138 \mathrm{~b}$ are largely invariant to changes in the peptide sequence but closely intertwined with the particular structural template the compound interacts with. The preferred modes of interaction with $\beta$-sheet rich fibrillar oligomers are mainly directed toward hydrophobic patches on the surface of the aggregates, whereas anle $138 \mathrm{~b}$ interactions with disordered peptide aggregates involve the canonical peptide backbone structure. The substantial desolvation of the small molecule upon binding appears to be crucial to establish stable hydrogen bond interactions to peptide backbones within the aggregates. Moreover, our findings on the molecular geometry and concomitant effects on intermolecular interactions for the inactive compound anle234b underscore and corroborate the specificity of anle138b's mode of inhibition directed toward oligomers formed by aggregation-prone peptides. From the above presented results we infer that anle $138 \mathrm{~b}$ is able to significantly reduce the amount of intermolecular hydrogen bonding. Thereby, anle138b blocks the path toward extended $\beta$-strands already at the level of the dimer and furthermore obstructs the formation of fibril-like structure elements in higher order oligomers. Altogether the atomistic description of the anle $138 \mathrm{~b}$ binding process offers a compelling rationale for the modulation of oligomeric structures as shown by experiments. We thus expect that the detailed information on inhibition of oligomer aggregate formation by the small molecule anle138b will contribute to and will advance the ongoing efforts of rational drug development.

\section{METHODS}

Simulation Protocol. The GROMACS simulation software package (version 4.6.7) ${ }^{100,101}$ was used to set up, carry out, and analyze the MD simulations. The particle-mesh-Ewald (PME) ${ }^{102,103}$ method was used to treat long-ranged electrostatic interactions at every step with a grid spacing of $0.1 \mathrm{~nm}$, and the relative tolerance at the cutoff was set to $10^{-6}$. Periodic boundary conditions were applied to the simulation box.

MD Simulations. After system preparation, an energy minimization using the steepest descent algorithm was performed (1000 steps). Subsequently, a $10 \mathrm{ps}$ run with position restraints on the protein backbone atoms was carried out to equilibrate the system. The Berendsen barostat ${ }^{104}$ was used to keep the pressure constant $(1 \mathrm{bar})$. The velocity-rescaling ${ }^{105}$ algorithm was applied to couple the system to an external heat bath with a temperature of $300 \mathrm{~K}$ using a time constant of $\tau=0.1 \mathrm{ps}$. Initial velocities were taken according to the Maxwell-Boltzmann distribution at $300 \mathrm{~K}$. For production runs, the pressure coupling scheme of Parrinello-Rahman ${ }^{106}$ was used to keep the system at a pressure of $1 \mathrm{bar}(\tau=2.5 \mathrm{ps})$. MD simulations with and without small molecule were performed employing the AMBER99SB*-ILDN ${ }^{84,85,87}$ force field together with the TIP3P water model. ${ }^{86}$ Bonds between all atoms in protein and ligand were constrained using the P-LINCS ${ }^{107}$ algorithm. Water molecules were constrained using SETTLE. ${ }^{108}$ Hydrogen atoms were converted to virtual sites, ${ }^{109}$ and the integration time step was set to $4 \mathrm{fs}$. Simulations were performed with the native GPU accelerated version 4.6 of the GROMACS software package. ${ }^{101}$ A nonbonded Verlet scheme with cutoff for the van der Waals and electrostatic interactions together with a buffered pair-list was utilized. The cutoffs were set to $1.0 \mathrm{~nm}$. The neighbor lists for nonbonded interactions were updated every 40 steps.

Replica Exchange Simulations. The relative free energies of the anle138b conformers 1-4 (first tautomer) and 5-8 (second tautomer) in gas phase were estimated from the conformation populations sampled in temperature replica exchange molecular dynamics (REMD) simulations. In total, eight independent REMD simulations were performed, i.e., a topology of each conformer was used in one REMD simulation. The topologies of the conformers differ in the partial charge assignment only.

The temperature ladder of 8 values ranging from 300 to $1000 \mathrm{~K}$ was constructed using the approach described in ref 110 . Since the REMD simulations were performed in vacuum, a stochastic dynamics integrator with the friction constant of $0.5 \mathrm{ps}^{-1}$ was used. Infinite cutoff values were set for the nonbonded interactions. Each simulation was performed for $100 \mathrm{~ns}$ with the integration time-step of 2 fs. Finally, the population densities were extracted from the lowest temperature trajectory.

Free Energy Difference Between Anle138b Tautomers. To estimate the free energy difference between the tautomer pairs (conformers 1-5, 2-6, 3-7, and 4-8), we performed alchemical free energy calculations based on the nonequilibrium molecular dynamics simulations. First, hybrid structures and topologies for the ligands were built such that the physical end-states of an alchemical transition would represent one tautomer only. For that we used an in-house set of tools based on the pmx ${ }^{111}$ library. For every tautomer pair, we 
performed 10 independent $10 \mathrm{~ns}$ equilibrium simulations in vacuum for both tautomers separately. Subsequently, from each equilibrium simulation the first 2 ns were discarded and from the rest of the trajectory 80 frames were extracted equidistantly in time. The obtained snapshots were used to initiate nonequilibrium 20 ps alchemical transitions from the state $\mathrm{A}$ to $\mathrm{B}$ and vice versa (the states $\mathrm{A}$ and $\mathrm{B}$ represent the tautomers). The simulation parameters were the same as used for the REMD runs. For the alchemical transitions the nonbonded interactions were soft-cored. ${ }^{112}$ The work values required for the transitions to take place were collected and used to estimate the free energy difference between the tautomers based on the Crooks Fluctuation Theorem ${ }^{113}$ utilizing the maximum likelihood estimator. ${ }^{114}$

The procedure described above allowed calculating the free energy differences between the tautomers. However, the alchemical approach required replacing hydrogen atoms with the dummy particles during the transition from one tautomeric state to another. While the dummies were not felt by the rest of the system via nonbonded interactions, these particles were nevertheless bound to the molecule and their contribution to the free energy difference needed to be accounted for. To quantify this contribution, we disabled all the bonded interactions for the dummy atoms and calculated the potential energy of the molecule for every snapshot extracted from the equilibrium simulations. The same procedure was repeated without disabling the bonded dummy atom interactions. The calculated potential energy values were used to evaluate the change in free energy due to the dummy atom addition by means of the Zwanzig's Perturbation formula. ${ }^{115}$

Anle138b Solvation Free Energy Calculations in Water. The solvation free energy was calculated for every tautomer and every anle138b conformer. The nonequilibrium free energy calculation protocol, as described above, was employed. For the simulations we utilized Gromacs functionality of decoupling a ligand from its environment, at the same time retaining the intramolecular interactions. Most of the simulation parameters were identical to those used in the peptide simulations, except the following: for the equilibration runs a stochastic dynamics integrator with the friction constant of $0.5 \mathrm{ps}^{-1}$ was used. The integration time step was set to $2 \mathrm{fs}$ and the dispersion correction for energy and pressure was applied. The alchemical transitions in the case of solvation free energy calculations were performed in 50 ps using a leapfrog integrator with the velocity rescaling thermostat (time constant of $0.1 \mathrm{ps}$ ). During the alchemical transitions soft-coring was applied to the nonbonded interactions.

Anle138b Solvation Free Energy Calculations in 1-Octanol. The starting structure and force field parameters for 1-octanol were obtained from the virtual chemistry library. ${ }^{116}$ GAFF based parameters for 1-octanol were used. Prior to the free energy calculations, the simulation system was equilibrated in several stages. First, anle138b conformer 1 was placed in the box with 3531 -octanol molecules. An equilibrium simulation at $320 \mathrm{~K}$ was performed for $100 \mathrm{~ns}$. Afterward, anle138b molecule was removed from the box and the pure 1-octanol box was further equilibrated for $100 \mathrm{~ns}$ at $320 \mathrm{~K}$ temperature. The final configuration of this simulation was used to initiate calculations of solvation free energies of the anle $138 \mathrm{~b}$ conformers following the same procedure as for the hydration free energies. The simulation parameters were the same as for the hydration free energy calculations, except for the higher temperature of $320 \mathrm{~K}$, equilibrium simulation length (50 ns) and longer alchemical transitions (200 ps).

Analysis Protocol. From the individual simulation trajectories, samples were collected every 100 ps and used throughout subsequent analyses. After pooling all trajectories of each peptide sequence, a pairwise contact analysis was carried out to identify the individual oligomer sizes, containing two to four chains. Peptides that shared an interchain contact of the main chain atoms within a distance of 0.45 $\mathrm{nm}$ were considered to be part the same aggregate.

Principal Component Analysis and Conformational Clustering of Aggregate Structures. A principal component analysis (PCA $)^{117}$ was carried out on the main chain atomic coordinates of the dimeric aggregation state for each sequence. Prior to the construction and diagonalization of the covariance matrix of atomic displacements, conformations from all trajectories (in the presence and absence of anle138b) were concatenated and subsequently superimposed using the variance minimization method. ${ }^{118}$ The corresponding first and second eigenvectors used for the projections of the individual conformational ensembles were derived by the construction and diagonalization of the covariance matrix of atomic displacements. To obtain a representative structural subensemble, a conformational clustering was performed on the projected set of structures using the $\mathrm{k}$-means Hartigan-Wong algorithm ${ }^{119}$ implemented in the statistical software package $\mathrm{R}^{120}$ Cluster centers were selected according to the global k-means algorithm. ${ }^{121}$ Structures with the smallest root-meansquare deviation (RMSD) to the geometrical centers of the clusters were selected as representatives. To identify dominant substates within each conformational cluster obtained by k-means clustering, an additional cluster analysis based on the Daura algorithm ${ }^{122}$ was performed. After evaluating the pairwise RMSD of the peptide main chain atoms of all aggregate conformations within a given pool of structures, those structures with RMSD values smaller than $0.15 \mathrm{~nm}$ were grouped into a common substate. The consecutive clustering approach allowed a fine-grained analysis of the conformational space, where the results of the k-means clustering were used to calculate the population frequency among the various conformational states. The subsequent clustering into substates using the Daura algorithm permitted to assess the dynamical sampling of each k-means cluster. The total numbers of conformational clusters obtained for the simulations of dimeric aggregates were as follows (k-means/minimum RMSD neighbor substates): hTau40 ${ }_{305-314},(24 / 73) ; \mathrm{A} \beta_{14-23},(25 /$ 83); hIAPP $20-29,(21 / 138)$; Sup $35 \mathrm{~N}_{7-16},(22 / 120)$.

\section{ASSOCIATED CONTENT}

\section{S Supporting Information}

The Supporting Information is available free of charge on the ACS Publications website at DOI: 10.1021/acschemneuro.7b00325.

Example of hTau40 dimer d05-314 $_{3}$ simulation $(10 \mu \mathrm{s})$ (AVI)

Example of hTau40 dimer d05-314 simulation with anle138b $(7 \mu \mathrm{s})$ (AVI)

Description of simulation methods and data analysis, additional Figures and Tables (PDF)

\section{AUTHOR INFORMATION}

\section{Corresponding Author}

*E-mail: bgroot@gwdg.de. Phone: ++49-551-2012308. Fax: + +49-551-2012302.

ORCID $\odot$

Vytautas Gapsys: 0000-0002-6761-7780

Bert L. de Groot: 0000-0003-3570-3534

\section{Author Contributions}

D.M., V.G., C.G., and B.L.d.G conceived the experiments. D.M. and V.G. performed the experiments and analyzed the results. All authors contributed to discussions and wrote the manuscript.

Notes

The authors declare no competing financial interest.

\section{ACKNOWLEDGMENTS}

The simulations presented in this work were in part carried out on the HYDRA supercomputer. We would like to acknowledge the computer centre Garching (RZG) for providing the necessary computational resources. We are grateful to Armin Giese, Andrei Leonov, and Sergey Ryazanov for helpful discussions and critical reading of the manuscript and thank Petra Kellers for editorial assistance. 


\section{REFERENCES}

(1) The Lancet Neurology (2016) Finding a cure for Alzheimer's disease starts with prevention. Lancet Neurol. 15, 649.

(2) Wischik, C. M., Harrington, C. R., and Storey, J. M. (2014) Tauaggregation inhibitor therapy for Alzheimer's disease. Biochem. Pharmacol. 88, 529-539.

(3) Callaway, E. (2012) Alzheimer's drugs take a new tack. Nature $489,13-14$.

(4) Cummings, J. L., Morstorf, T., and Zhong, K. (2014) Alzheimer's disease drug-development pipeline: few candidates, frequent failures. Alzheimer's Res. Ther. 6, 37.

(5) Caughey, B., and Lansbury, P. (2003) Protofibrils, pores, fibrils, and neurodegeneration: separating the responsible protein aggregates from the innocent bystanders. Annu. Rev. Neurosci. 26, 267-298.

(6) Haass, C., and Selkoe, D. J. (2007) Soluble protein oligomers in neurodegeneration: lessons from the Alzheimer's amyloid $\beta$-peptide. Nat. Rev. Mol. Cell Biol. 8, 101-112.

(7) Chiti, F., and Dobson, C. M. (2006) Protein misfolding, functional amyloid, and human disease. Annu. Rev. Biochem. 75, 333366.

(8) Eisenberg, D., and Jucker, M. (2012) The Amyloid State of Proteins in Human Diseases. Cell 148, 1188-1203.

(9) Kayed, R., Head, E., Thompson, J. L., McIntire, T. M., Milton, S. C., Cotman, C. W., and Glabe, C. G. (2003) Common Structure of Soluble Amyloid Oligomers Implies Common Mechanism of Pathogenesis. Science 300, 486-489.

(10) Glabe, C. G. (2008) Structural classification of toxic amyloid oligomers. J. Biol. Chem. 283, 29639-29643.

(11) Fändrich, M. (2012) Oligomeric Intermediates in Amyloid Formation: Structure Determination and Mechanisms of Toxicity. J. Mol. Biol. 421, 427-440.

(12) Benilova, I., Karran, E., and De Strooper, B. (2012) The toxic $\mathrm{A} \beta$ oligomer and Alzheimer's disease: an emperor in need of clothes. Nat. Neurosci. 15, 349-357.

(13) Necula, M., Kayed, R., Milton, S., and Glabe, C. G. (2007) Small Molecule Inhibitors of Aggregation Indicate That Amyloid $\beta$ Oligomerization and Fibrillization Pathways Are Independent and Distinct. J. Biol. Chem. 282, 10311-10324.

(14) Bateman, R. (2015) Alzheimer's disease and other dementias: advances in 2014. Lancet Neurol. 14, 4-6.

(15) Bulic, B., Pickhardt, M., and Mandelkow, E. (2013) Progress and Developments in Tau Aggregation Inhibitors for Alzheimer Disease. J. Med. Chem. 56, 4135-4155.

(16) Toth, G., et al. (2014) Targeting the Intrinsically Disordered Structural Ensemble of $\alpha$-Synuclein by Small Molecules as a Potential Therapeutic Strategy for Parkinson's Disease. PLoS One 9, e87133.

(17) Citron, M. (2010) Alzheimer's disease: strategies for disease modification. Nat. Rev. Drug Discovery 9, 387-398.

(18) Eisele, Y. S., Monteiro, C., Fearns, C., Encalada, S. E., Wiseman, R. L., Powers, E. T., and Kelly, J. W. (2015) Targeting protein aggregation for the treatment of degenerative diseases. Nat. Rev. Drug Discovery 14, 759-780.

(19) Young, L. M., Saunders, J. C., Mahood, R. A., Revill, C. H., Foster, R. J., Tu, L.-H., Raleigh, D. P., Radford, S. E., and Ashcroft, A.E. (2015) Screening and classifying small molecule inhibitors of amyloid formation using ion mobility spectrometry-mass spectrometry. Nat. Chem. 7, 73-81.

(20) Cremades, N., Cohen, S., Deas, E., Abramov, A., Chen, A., Orte, A., Sandal, M., Clarke, R., Dunne, P., Aprile, F., Bertoncini, C., Wood, N., Knowles, T., Dobson, C., and Klenerman, D. (2012) Direct Observation of the Interconversion of Normal and Toxic Forms of $\alpha$ Synuclein. Cell 149, 1048-1059.

(21) Chen, S. W., et al. (2015) Structural characterization of toxic oligomers that are kinetically trapped during $\alpha$-Synuclein fibril formation. Proc. Natl. Acad. Sci. U. S. A. 112, E1994-E2003.

(22) Shammas, S. L., Garcia, G. A., Kumar, S., Kjaergaard, M., Horrocks, M. H., Shivji, N., Mandelkow, E., Knowles, T. P., Mandelkow, E., and Klenerman, D. (2015) A mechanistic model of tau amyloid aggregation based on direct observation of oligomers. Nat. Commun. 6, 7025.

(23) Abedini, A., Plesner, A., Cao, P., et al. (2016) Time-resolved studies define the nature of toxic IAPP intermediates, providing insight for anti-amyloidosis therapeutics. eLife 5, e12977.

(24) Seo, J., Hoffmann, W., Warnke, S., Huang, X., Gewinner, S., Schöllkopf, W., Bowers, M. T., von Helden, G., and Pagel, K. (2017) An infrared spectroscopy approach to follow $\beta$-sheet formation in peptide amyloid assemblies. Nat. Chem. 9, 39-44.

(25) Do, T. D., LaPointe, N. E., Nelson, R., Krotee, P., Hayden, E. Y., Ulrich, B., Quan, S., Feinstein, S. C., Teplow, D. B., Eisenberg, D., Shea, J.-E., and Bowers, M. T. (2016) Amyloid $\beta$-Protein C-Terminal Fragments: Formation of Cylindrins and $\beta$-Barrels. J. Am. Chem. Soc. $138,549-557$.

(26) Kreutzer, A. G., Yoo, S., Spencer, R. K., and Nowick, J. S. (2017) Stabilization, Assembly, and Toxicity of Trimers Derived from A $\beta$. J. Am. Chem. Soc. 139, 966-975.

(27) Laganowsky, A., Liu, C., Sawaya, M., Whitelegge, J., Park, J., Zhao, M., Pensalfini, A., Soriaga, A., Landau, M., Teng, P., Cascio, D., Glabe, C., and Eisenberg, D. (2012) Atomic view of a toxic amyloid small oligomer. Science 335, 1228-1231.

(28) Lendel, C., Bjerring, M., Dubnovitsky, A., Kelly, R. T., Filippov, A., Antzutkin, O. N., Nielsen, N. C., and Härd, T. (2014) A Hexameric Peptide Barrel as Building Block of Amyloid- $\beta$ Protofibrils. Angew. Chem., Int. Ed. 53, 12756-12760.

(29) Doig, A. J., del Castillo-Frias, M. P., Berthoumieu, O., Tarus, B., Nasica-Labouze, J., Sterpone, F., Nguyen, P. H., Hooper, N. M., Faller, P., and Derreumaux, P. (2017) Why Is Research on Amyloid- $\beta$ Failing to Give New Drugs for Alzheimer's Disease? ACS Chem. Neurosci. 8, 1435-1437.

(30) Doig, A. J., and Derreumaux, P. (2015) Inhibition of protein aggregation and amyloid formation by small molecules. Curr. Opin. Struct. Biol. 30, 50-56.

(31) Granata, D., Baftizadeh, F., Habchi, J., Galvagnion, C., De Simone, A., Camilloni, C., Laio, A., and Vendruscolo, M. (2015) The inverted free energy landscape of an intrinsically disordered peptide by simulations and experiments. Sci. Rep. 5, 15449.

(32) Levine, Z. A., and Shea, J.-E. (2017) Simulations of disordered proteins and systems with conformational heterogeneity. Curr. Opin. Struct. Biol. 43, 95-103.

(33) Baftizadeh, F., Biarnes, X., Pietrucci, F., Affinito, F., and Laio, A. (2012) Multidimensional View of Amyloid Fibril Nucleation in Atomistic Detail. J. Am. Chem. Soc. 134, 3886-3894.

(34) Matthes, D., Gapsys, V., Brennecke, J. T., and de Groot, B. L. (2016) An Atomistic View of Amyloidogenic Self-assembly: Structure and Dynamics of Heterogeneous Conformational States in the Prenucleation Phase. Sci. Rep. 6, 33156.

(35) Nasica-Labouze, J., et al. (2015) Amyloid beta Protein and Alzheimer's Disease: When Computer Simulations Complement Experimental Studies. Chem. Rev. 115, 3518-3563.

(36) Straub, J. E., and Thirumalai, D. (2011) Toward a Molecular Theory of Early and Late Events in Monomer to Amyloid Fibril Formation. Annu. Rev. Phys. Chem. 62, 437-463.

(37) Rauscher, S., Gapsys, V., Gajda, M. J., Zweckstetter, M., de Groot, B. L., and Grubmüller, H. (2015) Structural Ensembles of Intrinsically Disordered Proteins Depend Strongly on Force Field: A Comparison to Experiment. J. Chem. Theory Comput. 11, 5513-5524.

(38) Piana, S., Donchev, A. G., Robustelli, P., and Shaw, D. E. (2015) Water Dispersion Interactions Strongly Influence Simulated Structural Properties of Disordered Protein States. J. Phys. Chem. B 119, 51135123.

(39) Ganguly, P., Do, T. D., Larini, L., LaPointe, N. E., Sercel, A. J., Shade, M. F., Feinstein, S. C., Bowers, M. T., and Shea, J.-E. (2015) Tau Assembly: The Dominant Role of PHF6 (VQIVYK) in Microtubule Binding Region Repeat R3. J. Phys. Chem. B 119, $4582-4593$.

(40) Lemkul, J. A., and Bevan, D. R. (2012) The Role of Molecular Simulations in the Development of Inhibitors of Amyloid $\beta$-Peptide 
Aggregation for the Treatment of Alzheimer's Disease. ACS Chem. Neurosci. 3, 845-856.

(41) Ehrnhoefer, D. E., Bieschke, J., Boeddrich, A., Herbst, M., Masino, L., Lurz, R., Engemann, S., Pastore, A., and Wanker, E. E. (2008) EGCG redirects amyloidogenic polypeptides into unstructured, off-pathway oligomers. Nat. Struct. Mol. Biol. 15, 558-566.

(42) Wobst, H. J., Sharma, A., Diamond, M. I., Wanker, E. E., and Bieschke, J. (2015) The green tea polyphenol (-)-epigallocatechin gallate prevents the aggregation of tau protein into toxic oligomers at substoichiometric ratios. FEBS Lett. 589, 77-83.

(43) Bleiholder, C., Do, T. D., Wu, C., Economou, N. J., Bernstein, S. S., Buratto, S. K., Shea, J.-E., and Bowers, M. T. (2013) Ion Mobility Spectrometry Reveals the Mechanism of Amyloid Formation of A $\beta(25-35)$ and Its Modulation by Inhibitors at the Molecular Level: Epigallocatechin Gallate and Scyllo-inositol. J. Am. Chem. Soc. 135, 16926-16937.

(44) Wang, H., and Raleigh, D. P. (2014) General Amyloid Inhibitors? A Critical Examination of the Inhibition of IAPP Amyloid Formation by Inositol Stereoisomers. PLoS One 9, e104023.

(45) McLaurin, J., Golomb, R., Jurewicz, A., Antel, J. P., and Fraser, P. E. (2000) Inositol Stereoisomers Stabilize an Oligomeric Aggregate of Alzheimer Amyloid $\beta$ Peptide and Inhibit A $\beta$-induced Toxicity. J. Biol. Chem. 275, 18495-18502.

(46) McLaurin, J., et al. (2006) Cyclohexanehexol inhibitors of $\mathrm{A} \beta$ aggregation prevent and reverse Alzheimer phenotype in a mouse model. Nat. Med. 12, 801-808.

(47) Li, G., Rauscher, S., Baud, S., and Pomes, R. (2012) Binding of Inositol Stereoisomers To Model Amyloidogenic Peptides. J. Phys. Chem. B 116, 1111-1119.

(48) Li, G., and Pomes, R. (2013) Binding Mechanism of Inositol Stereoisomers to Monomers and Aggregates of $\mathrm{A} \beta(16-22)$. J. Phys. Chem. B 117, 6603-6613.

(49) Zhang, T., Zhang, J., Derreumaux, P., and Mu, Y. (2013) Molecular Mechanism of the Inhibition of EGCG on the Alzheimer A 1 1-42 Dimer. J. Phys. Chem. B 117, 3993-4002.

(50) Bertsch, U., Winklhofer, K. F., Hirschberger, T., Bieschke, J., Weber, P., Hartl, F. U., Tavan, P., Tatzelt, J., Kretzschmar, H. A., and Giese, A. (2005) Systematic Identification of Antiprion Drugs by High-Throughput Screening Based on Scanning for Intensely Fluorescent Targets. J. Virol. 79, 7785-7791.

(51) Wagner, J., et al. (2013) Anle138b: a novel oligomer modulator for disease-modifying therapy of neurodegenerative diseases such as prion and Parkinson's disease. Acta Neuropathol. 125, 795-813.

(52) Deeg, A. A., Reiner, A. M., Schmidt, F., Schueder, F., Ryazanov, S., Ruf, V. C., Giller, K., Becker, S., Leonov, A., Griesinger, C., Giese, A., and Zinth, W. (2015) Anle138b and related compounds are aggregation specific fluorescence markers and reveal high affinity binding to $\alpha$-synuclein aggregates. Biochim. Biophys. Acta, Gen. Subj. 1850, 1884-1890.

(53) Wagner, J., et al. (2015) Reducing tau aggregates with anle138b delays disease progression in a mouse model of tauopathies. Acta Neuropathol. 130, 619-631.

(54) Schmidt, F., Levin, J., Kamp, F., Kretzschmar, H., Giese, A., and Bötzel, K. (2012) Single-Channel Electrophysiology Reveals a Distinct and Uniform Pore Complex Formed by $\alpha$-Synuclein Oligomers in Lipid Membranes. PLoS One 7, e42545.

(55) von Bergen, M., Friedhoff, P., Biernat, J., Heberle, J., Mandelkow, E.-M., and Mandelkow, E. (2000) Assembly of $\tau$ protein into Alzheimer paired helical filaments depends on a local sequence motif (306VQIVYK311) forming $\beta$ structure. Proc. Natl. Acad. Sci. U. S. A. $97,5129-5134$.

(56) Sawaya, M. R., Sambashivan, S., Nelson, R., Ivanova, M. I., Sievers, S. A., Apostol, M. I., Thompson, M. J., Balbirnie, M., Wiltzius, J. J. W., McFarlane, H. T., Madsen, A. O., Riekel, C., and Eisenberg, D. (2007) Atomic structures of amyloid cross-beta spines reveal varied steric zippers. Nature 447, 453-457.

(57) Daebel, V., Chinnathambi, S., Biernat, J., Schwalbe, M., Habenstein, B., Loquet, A., Akoury, E., Tepper, K., Mueller, H., Baldus, M., Griesinger, C., Zweckstetter, M., Mandelkow, E., Vijayan,
V., and Lange, A. (2012) $\beta$-Sheet Core of Tau Paired Helical Filaments Revealed by Solid-State NMR. J. Am. Chem. Soc. 134, 13982-13989.

(58) Colletier, J.-P., Laganowsky, A., Landau, M., Zhao, M., Soriaga, A. B., Goldschmidt, L., Flot, D., Cascio, D., Sawaya, M. R., and Eisenberg, D. (2011) Molecular basis for amyloid-beta polymorphism. Proc. Natl. Acad. Sci. U. S. A. 108, 16938-16943.

(59) Colvin, M. T., Silvers, R., Ni, Q. Z., Can, T. V., Sergeyev, I., Rosay, M., Donovan, K. J., Michael, B., Wall, J., Linse, S., and Griffin, R. G. (2016) Atomic Resolution Structure of Monomorphic A $\beta 42$ Amyloid Fibrils. J. Am. Chem. Soc. 138, 9663-9674.

(60) Buchanan, L. E., Dunkelberger, E. B., Tran, H. Q., Cheng, P.-N., Chiu, C.-C., Cao, P., Raleigh, D. P., de Pablo, J. J., Nowick, J. S., and Zanni, M. T. (2013) Mechanism of IAPP amyloid fibril formation involves an intermediate with a transient $\beta$-sheet. Proc. Natl. Acad. Sci. U. S. A. 110, 19285-19290.

(61) Westermark, P., Engström, U., Johnson, K., Westermark, G., and Betsholtz, C. (1990) Islet amyloid polypeptide: pinpointing amino acid residues linked to amyloid fibril formation. Proc. Natl. Acad. Sci. U. S. A. 87, 5036-5040.

(62) Balbirnie, M., Grothe, R., and Eisenberg, D. S. (2001) An amyloid-forming peptide from the yeast prion Sup35 reveals a dehydrated $\beta$-sheet structure for amyloid. Proc. Natl. Acad. Sci. U. S. A. 98, 2375-2380.

(63) Krishnan, R., Goodman, J. L., Mukhopadhyay, S., Pacheco, C. D., Lemke, E. A., Deniz, A. A., and Lindquist, S. (2012) Conserved features of intermediates in amyloid assembly determine their benign or toxic states. Proc. Natl. Acad. Sci. U. S. A. 109, 11172-11177.

(64) Kabsch, W., and Sander, C. (1983) Dictionary of protein secondary structure: pattern recognition of hydrogen-bonded and geometrical features. Biopolymers 22, 2577-2637.

(65) Wald, A. (1943) Tests of statistical hypotheses concerning several parameters when the number of observations is large. Trans. Am. Math. Soc. 54, 426-482.

(66) Fricke, H., Gerlach, A., Unterberg, C., Wehner, M., Schrader, T., and Gerhards, M. (2009) Interactions of Small Protected Peptides with Aminopyrazole Derivatives: The Efficiency of Blocking a $\beta$-Sheet Model in the Gas Phase. Angew. Chem., Int. Ed. 48, 900-904.

(67) Wang, W., and Weisz, K. (2007) Characterization of PeptidePyrazole Interactions in Solution by Low-Temperature NMR Studies. Chem. - Eur. J. 13, 854-861.

(68) Rzepecki, P., Nagel-Steger, L., Feuerstein, S., Linne, U., Molt, O., Zadmard, R., Aschermann, K., Wehner, M., Schrader, T., and Riesner, D. (2004) Prevention of Alzheimer's Disease-associated A $\beta$ Aggregation by Rationally Designed Nonpeptidic $\beta$-Sheet Ligands. J. Biol. Chem. 279, 47497-47505.

(69) Rzepecki, P., and Schrader, T. (2005) $\beta$-Sheet Ligands in Action: KLVFF Recognition by Aminopyrazole Hybrid Receptors in Water. J. Am. Chem. Soc. 127, 3016-3025.

(70) Porat, Y., Abramowitz, A., and Gazit, E. (2006) Inhibition of Amyloid Fibril Formation by Polyphenols: Structural Similarity and Aromatic Interactions as a Common Inhibition Mechanism. Chem. Biol. Drug Des. 67, 27-37.

(71) Hochdörffer, K., März-Berberich, J., Nagel-Steger, L., Epple, M., Meyer-Zaika, W., Horn, A. H., Sticht, H., Sinha, S., Bitan, G., and Schrader, T. (2011) Rational Design of $\beta$-Sheet Ligands Against A $\beta 42$ Induced Toxicity. J. Am. Chem. Soc. 133, 4348-4358.

(72) Liu, C., Sawaya, M. R., Cheng, P.-N., Zheng, J., Nowick, J. S., and Eisenberg, D. (2011) Characteristics of Amyloid-Related Oligomers Revealed by Crystal Structures of Macrocyclic beta-Sheet Mimics. J. Am. Chem. Soc. 133, 6736-6744.

(73) Liu, C., Zhao, M., Jiang, L., Cheng, P.-N., Park, J., Sawaya, M. R, Pensalfini, A., Gou, D., Berk, A. J., Glabe, C. G., Nowick, J., and Eisenberg, D. (2012) Out-of-register beta-sheets suggest a pathway to toxic amyloid aggregates. Proc. Natl. Acad. Sci. U. S. A. 109, 2091320918.

(74) Soriaga, A. B., Sangwan, S., Macdonald, R., Sawaya, M. R., and Eisenberg, D. (2016) Crystal Structures of IAPP Amyloidogenic 
Segments Reveal a Novel Packing Motif of Out-of-Register Beta Sheets. J. Phys. Chem. B 120, 5810-5816.

(75) Ahmed, M., Davis, J., Aucoin, D., Sato, T., Ahuja, S., Aimoto, S., Elliott, J., Van Nostrand, W., and Smith, S. (2010) Structural conversion of neurotoxic amyloid- $\beta_{1-42}$ oligomers to fibrils. Nat. Struct. Mol. Biol. 17, 561-567.

(76) Stroud, J. C., Liu, C., Teng, P. K., and Eisenberg, D. (2012) Toxic fibrillar oligomers of amyloid- $\beta$ have cross- $\beta$ structure. Proc. Natl. Acad. Sci. U. S. A. 109, 7717-7722.

(77) Krotee, P., Rodriguez, J. A., Sawaya, M. R., et al. (2017) Atomic structures of fibrillar segments of hIAPP suggest tightly mated $\beta$-sheets are important for cytotoxicity. eLife 6, e19273.

(78) Celej, M. S., Sarroukh, R., Goormaghtigh, E., Fidelio, G. D., Ruysschaert, J.-M., and Raussens, V. (2012) Toxic prefibrillar $\alpha$ synuclein amyloid oligomers adopt a distinctive antiparallel $\beta$-sheet structure. Biochem. J. 443, 719-726.

(79) Matthes, D., Gapsys, V., and de Groot, B. L. (2012) Driving Forces and Structural Determinants of Steric Zipper Peptide Oligomer Formation Elucidated by Atomistic Simulations. J. Mol. Biol. 421, 390416.

(80) De Simone, A., and Derreumaux, P. (2010) Low molecular weight oligomers of amyloid peptides display beta-barrel conformations: A replica exchange molecular dynamics study in explicit solvent. J. Chem. Phys. 132, 165103.

(81) Riccardi, L., Nguyen, P. H., and Stock, G. (2012) Construction of the Free Energy Landscape of Peptide Aggregation from Molecular Dynamics Simulations. J. Chem. Theory Comput. 8, 1471-1479.

(82) Matthes, D., Gapsys, V., Daebel, V., and de Groot, B. L. (2011) Mapping the Conformational Dynamics and Pathways of Spontaneous Steric Zipper Peptide Oligomerization. PLoS One 6, e19129.

(83) Hwang, W., Zhang, S., Kamm, R. D., and Karplus, M. (2004)

Kinetic control of dimer structure formation in amyloid fibrillogenesis. Proc. Natl. Acad. Sci. U. S. A. 101, 12916-12921.

(84) Best, R. B., and Hummer, G. (2009) Optimized molecular dynamics force fields applied to the helix-coil transition of polypeptides. J. Phys. Chem. B 113, 9004-9015.

(85) Lindorff-Larsen, K., Piana, S., Palmo, K., Maragakis, P., Klepeis, J. L., Dror, R. O., and Shaw, D. E. (2010) Improved side-chain torsion potentials for the Amber ff99SB protein force field. Proteins: Struct., Funct., Genet. 78, 1950-1958.

(86) Jorgensen, W. L., Chandrasekhar, J., Madura, J. D., Impey, R. W., and Klein, M. L. (1983) Comparison of Simple Potential Functions for Simulating Liquid Water. J. Chem. Phys. 79, 926.

(87) Hornak, V., Abel, R., Okur, A., Strockbine, B., Roitberg, A., and Simmerling, C. (2006) Comparison of multiple Amber force fields and development of improved protein backbone parameters. Proteins: Struct., Funct., Genet. 65, 712-725.

(88) Lindorff-Larsen, K., Maragakis, P., Piana, S., Eastwood, M. P., Dror, R. O., and Shaw, D. E. (2012) Systematic Validation of Protein Force Fields against Experimental Data. PLoS One 7, e32131.

(89) Sangwan, S., et al. (2017) Atomic structure of a toxic, oligomeric segment of SOD1 linked to amyotrophic lateral sclerosis (ALS). Proc. Natl. Acad. Sci. U. S. A. 114, 8770-8775.

(90) Apostol, M. I., Perry, K., and Surewicz, W. K. (2013) Crystal Structure of a Human Prion Protein Fragment Reveals a Motif for Oligomer Formation. J. Am. Chem. Soc. 135, 10202-10205.

(91) Liu, P., Reed, M., Kotilinek, L., Grant, M., Forster, C., Qiang, W., Shapiro, S., Reichl, J., Chiang, A., Jankowsky, J., Wilmot, C., Cleary, J., Zahs, K., and Ashe, K. (2015) Quaternary Structure Defines a Large Class of Amyloid- $\beta$ Oligomers Neutralized by Sequestration. Cell Rep. 11, 1760-1771.

(92) Yan, L.-M., Velkova, A., Tatarek-Nossol, M., Andreetto, E., and Kapurniotu, A. (2007) IAPP Mimic Blocks A $\beta$ Cytotoxic SelfAssembly: Cross-Suppression of Amyloid Toxicity of $\mathrm{A} \beta$ and IAPP Suggests a Molecular Link between Alzheimer's Disease and Type II. Angew. Chem., Int. Ed. 46, 1246-1252.

(93) Sciarretta, K. L., Gordon, D. J., and Meredith, S. C. (2006) Peptide-Based Inhibitors of Amyloid Assembly. Methods Enzymol. 413, 273-312 Amyloid, Prions, and Other Protein Aggregates, Part C.
(94) Zheng, J., Liu, C., Sawaya, M. R., Vadla, B., Khan, S., Woods, R. J., Eisenberg, D., Goux, W. J., and Nowick, J. S. (2011) Macrocyclic beta-Sheet Peptides That Inhibit the Aggregation of a Tau-ProteinDerived Hexapeptide. J. Am. Chem. Soc. 133, 3144-3157.

(95) Wiltzius, J. J., Sievers, S. A., Sawaya, M. R., Cascio, D., Popov, D., Riekel, C., and Eisenberg, D. (2008) Atomic structure of the cross$\beta$ spine of islet amyloid polypeptide (amylin). Protein Sci. 17, 14671474.

(96) Habchi, J., Arosio, P., Perni, M., Costa, A. R., Yagi-Utsumi, M., Joshi, P., Chia, S., Cohen, S. I. A., Müller, M. B. D., Linse, S., Nollen, E. A. A., Dobson, C. M., Knowles, T. P. J., and Vendruscolo, M. (2016) An anticancer drug suppresses the primary nucleation reaction that initiates the production of the toxic $A \beta 42$ aggregates linked with Alzheimer's disease. Sci. Adv. 2, e1501244.

(97) Cohen, S. I. A., Linse, S., Luheshi, L. M., Hellstrand, E., White, D. A., Rajah, L., Otzen, D. E., Vendruscolo, M., Dobson, C. M., and Knowles, T. P. J. (2013) Proliferation of amyloid- $\beta 42$ aggregates occurs through a secondary nucleation mechanism. Proc. Natl. Acad. Sci. U. S. A. 110, 9758-9763.

(98) Herrmann, U. S., Schutz, A. K., Shirani, H., et al. (2015) Structure-based drug design identifies polythiophenes as antiprion compounds. Sci. Transl. Med. 7, 299ra123.

(99) Landau, M., Sawaya, M. R., Faull, K. F., Laganowsky, A., Jiang, L., Sievers, S. A., Liu, J., Barrio, J. R., and Eisenberg, D. (2011) Towards a Pharmacophore for Amyloid. PLoS Biol. 9, e1001080.

(100) Hess, B., Kutzner, C., Van Der Spoel, D., and Lindahl, E. (2008) GROMACS 4.0: algorithms for highly efficient, load-balanced, and scalable molecular simulation. J. Chem. Theory Comput. 4, 435447.

(101) Pronk, S., Pall, S., Schulz, R., Larsson, P., Bjelkmar, P., Apostolov, R., Shirts, M. R., Smith, J. C., Kasson, P. M., van der Spoel, D., Hess, B., and Lindahl, E. (2013) GROMACS 4.5: a highthroughput and highly parallel open source molecular simulation toolkit. Bioinformatics 29, 845-854.

(102) Darden, T., York, D., and Pedersen, L. (1993) Particle mesh Ewald: An $\mathrm{N}-\log (\mathrm{N})$ method for Ewald sums in large systems. J. Chem. Phys. 98, 10089-10092.

(103) Essmann, U., Perera, L., Berkowitz, M. L., Darden, T., Lee, H., and Pedersen, L. G. (1995) A smooth particle mesh Ewald method. J. Chem. Phys. 103, 8577-8593.

(104) Berendsen, H. J. C., Postma, J. P. M., van Gunsteren, W. F., DiNola, A., and Haak, J. R. (1984) Molecular dynamics with coupling to an external bath. J. Chem. Phys. 81, 3684-3690.

(105) Bussi, G., Donadio, D., and Parrinello, M. (2007) Canonical sampling through velocity rescaling. J. Chem. Phys. 126, 014101.

(106) Parrinello, M., and Rahman, A. (1981) Polymorphic transitions in single crystals: A new molecular dynamics method. J. Appl. Phys. 52, 7182 .

(107) Hess, B. (2008) P-LINCS: a parallel linear constraint solver for molecular simulation. J. Chem. Theory Comput. 4, 116-122.

(108) Miyamoto, S., and Kollman, P. A. (1992) Settle: An analytical version of the SHAKE and RATTLE algorithm for rigid water models. J. Comput. Chem. 13, 952-962.

(109) Feenstra, K. A., Hess, B., and Berendsen, H. J. C. (1999) Improving efficiency of large time-scale molecular dynamics simulations of hydrogen-rich systems. J. Comput. Chem. 20, 786-798.

(110) Patriksson, A., and van der Spoel, D. (2008) A temperature predictor for parallel tempering simulations. Phys. Chem. Chem. Phys. 10, 2073-2077.

(111) Gapsys, V., Michielssens, S., Seeliger, D., and de Groot, B. L. (2015) pmx: Automated protein structure and topology generation for alchemical perturbations. J. Comput. Chem. 36, 348-354.

(112) Gapsys, V., Seeliger, D., and de Groot, B. L. (2012) New softcore potential function for molecular dynamics based alchemical free energy calculations. J. Chem. Theory Comput. 8, 2373-2382.

(113) Crooks, G. E. (1999) Entropy production fluctuation theorem and the nonequilibrium work relation for free energy differences. Phys. Rev. E: Stat. Phys., Plasmas, Fluids, Relat. Interdiscip. Top. 60, 27212726. 
(114) Shirts, M. R., Bair, E., Hooker, G., and Pande, V. S. (2003) Equilibrium free energies from nonequilibrium measurements using maximum-likelihood methods. Phys. Rev. Lett. 91, 140601.

(115) Zwanzig, R. W. (1954) High-Temperature Equation of State by a Perturbation Method. J. Chem. Phys. 22, 1420-1426.

(116) Caleman, C., van Maaren, P. J., Hong, M., Hub, J. S., Costa, L. T., and van der Spoel, D. (2012) Force field benchmark of organic liquids: density, enthalpy of vaporization, heat capacities, surface tension, isothermal compressibility, volumetric expansion coefficient, and dielectric constant. J. Chem. Theory Comput. 8, 61-74.

(117) Amadei, A., Linssen, A. B. M., and Berendsen, H. J. C. (1993) Essential dynamics of proteins. Proteins: Struct., Funct., Genet. 17, 412425 .

(118) Gapsys, V., and de Groot, B. L. (2013) Optimal Superpositioning of Flexible Molecule Ensembles. Biophys. J. 104, 196-207. (119) Hartigan, J., and Wong, M. (1979) Algorithm AS 136: A Kmeans clustering algorithm. Appl. Stat. 28, 100-108.

(120) R Development Core Team (2010) R: A Language and Environment for Statistical Computing, R Foundation for Statistical Computing, Vienna, Austria.

(121) Likas, A., Vlassis, N., and Verbeek, J. J. (2003) The global kmeans clustering algorithm. Pattern Recogn. 36, 451-461.

(122) Daura, X., Gademann, K., Jaun, B., Seebach, D., van Gunsteren, W., and Mark, A. (1999) Peptide Folding: When Simulation Meets Experiment. Angew. Chem., Int. Ed. 38, 236-240. 